\title{
CÁLCULO DO FLUXO DE HARMÔNICOS EM SISTEMAS DE POTÊNCIA TRIFÁSICOS UTILIZANDO O MÉTODO DE INJEÇÃO DE CORRENTES COM SOLUÇÃO ITERATIVA
}

\author{
Abilio Manuel Variz \\ abilio@ieee.org \\ Sandoval Carneiro Jr. ${ }^{\dagger}$ \\ sandovalecoep.ufrj.br
}

\author{
José Luiz R. Pereira* \\ jluiz@ieee.org \\ Pedro G. Barbosa* \\ pedro.gomeseufjf.edu.br
}

\author{
*Universidade Federal de Juiz de Fora \\ Faculdade de Engenharia \\ Juiz de Fora, MG, CEP 36015-400, Brasil \\ ${ }^{\dagger}$ COPPE-Universidade Federal do Rio de Janeiro \\ Programa de Engenharia Elétrica \\ Caixa Postal 68504 \\ Rio de Janeiro, RJ, 21945-970, Brasil
}

\section{RESUMO}

Este trabalho apresenta uma metodologia, baseada nas equações de injeção de correntes (MICT), para o cálculo do fluxo de potência harmônico trifásico em sistemas elétricos equilibrados e desequilibrados (MICTH). A metodologia desenvolvida emprega o método iterativo de Newton-Raphson para obter a solução do sistema de equações não-lineares, onde o sistema matricial é formado por blocos relativos às freqüências harmônicas investigadas. Dessa maneira é possível representar as interações entre as componentes harmônicas de diferentes freqüências. Em paralelo foram desenvolvidos modelos dos principais dispositivos lineares e não-lineares conectados aos sistemas elétricos de potência. O MICTH (Método de Injeção de Correntes Trifásicas Harmônicas) e os modelos de componentes foram implementados em C++, com programação orientada a objetos, e utilizando técnicas de programação esparsa. Os resultados das simulações, no

\footnotetext{
Artigo submetido em 21/09/2007

1a. Revisão em 29/10/2007

2a. Revisão em 24/01/2008

Aceito sob recomendação do Editor Associado Prof. Denis Vinicius Coury
}

domínio da freqüência, obtidos com o programa MICTH foram validados a partir de comparações com resultados de simulações, no domínio do tempo, obtidos com o programa ATP/EMTP (Alternative Transients Program of Electromagnetic Transients Program).

PALAVRAS-CHAVE: Fluxo de potência harmônico, Análise harmônica, Modelo harmônico, Simulação harmônica.

\section{ABSTRACT}

This work presents a methodology, based on the current injection equations (MICT), for the calculus of the harmonic power flow in three-phase, balanced and unbalanced, electric power systems (MICTH). The developed methodology uses the Newton-Raphson method to obtain the solution of a set of non-linear equations, where the matrix system is built in a blocked form for each harmonic frequency. This mathematical approach permits to represent the interaction between harmonic components of different frequencies. It was also developed models for the main linear and nonlinear devices connected to the electric power systems. The MICTH (Three-Phase Harmonic Current Injection Method) 
and the devices models were implemented in $\mathrm{C}++$ using object-oriented and sparse-matrix programming techniques. The simulation results of the MICTH, obtained in the frequency domain, were validated with simulation results of the ATP/EMTP (Alternative Transients Program of Electromagnetic Transients Program), obtained in the time domain.

KEYWORDS: Load flow analysis, Power system harmonics, Harmonic simulation, Harmonic modeling, Harmonic analysis.

\section{INTRODUÇÃO}

Nos últimos anos ferramentas para análise harmônica vêm se tornando essenciais para o estudo e o planejamento de sistemas elétricos de potência (SEP) (IEEE, 1983; IEEE, 1993). Essa necessidade pode ser explicada pelo crescente número de cargas não-lineares conectadas aos sistemas elétricos.

Cargas não-lineares são responsáveis por distorcer as correntes e tensões dos sistemas elétricos (Semlyen e Shlash, 2000). Portanto, elas reduzem a eficiência das redes, devido ao aumento das perdas elétricas provocadas pelo efeito pelicular, além de produzirem efeitos indesejáveis como: ressonâncias harmônicas, mau funcionamento de circuitos eletrônicos, interferências em sistemas de comunicação, além de vibrações, ruídos e fadiga mecânica em transformadores, reatores e motores (Dufeey e Stratford, 1989; Wagner et alii, 1993).

Como o objetivo de minimizar os efeitos dos harmônicos e manter os índices de qualidade de energia elétrica em níveis seguros e aceitáveis, as concessionárias de energia elétrica têm estabelecido limites máximos para as distorções harmônicas medidas em suas redes elétricas (Domijan, 1993). Dentro deste contexto, medidas corretivas como a utilização de filtros passivos e ativos, além de técnicas de multiplicação de fase (transformadores de conexão) têm sido adotadas para reduzir ou eliminar a propagação dos harmônicos pelas redes.

Estudos de propagação harmônica nos SEP são geralmente realizados a partir de simulações digitais onde são usados modelos representativos dos elementos e da topologia da rede. Nestas simulações são calculados os harmônicos gerados e suas propagações pelo sistema. É possível assim quantificar e mapear os níveis de distorção harmônica em vários pontos da rede, identificando os pontos críticos para a operação do sistema elétrico. Desse modo, uma ferramenta eficiente para análise harmônica requer a representação precisa das características lineares e não-lineares dos diversos componentes de rede, de maneira a permitir a simulação do comportamento destes dispositivos quando alimentado por tensões e correntes distorcidas.

As principais metodologias empregadas para análise harmô- nica são apresentadas por Arrillaga et alii (1985), Dommel (1986), Semlyen e Shlash (2000), IEEE (1996), Smith et alii (1998), Xia e Heydt (1982), Marinos et alii (1994) e Acha e Madrigal (2001). Destacam-se também as metodologias baseadas no domínio do tempo, originalmente empregadas para análise transitória, apresentadas por Kulicke (1979) e Dommel et alii (1986), bem como importantes contribuições realizadas por Arrillaga et alii (1997), Xu et alii (1991), Arrillaga et alii (2004), Lin et alii (2004) e Semlyen et alii (1988) no desenvolvimento de metodologias para cálculo de fluxo harmônico no domínio da frequiência através de métodos iterativos de solução matemática. Vale ressaltar também importantes contribuições no desenvolvimento de modelos representativos de cargas não-lineares apresentados por Arrillaga et alii (1997) e Lima et alii (2003), e também modelos de transformadores dos quais se destacam os apresentados por Dommel et alii (1986), Dugui e Zheng (1998), Medina e Arrillaga (1992), Acha et alii (1989), Neves e Dommel (1993), Semlyen et alii (1987), Dommel et alii (1986), De Leon e Semlyen (1986) e Chen e Venkata (1997).

O principal objetivo deste trabalho é apresentar uma metodologia no domínio da freqüência para o cálculo de fluxo de potência harmônico trifásico em sistemas elétricos equilibrados e desequilibrados. A metodologia proposta é baseada nas equações de injeção de correntes e é denominada MICTH. A modelagem do sistema elétrico é feita através de vários subsistemas, um para cada freqüência harmônica de interesse, os quais são representados por estruturas de blocos no sistema matricial. Dessa maneira é possível representar as interações entre componentes harmônicos de diferentes freqüências. Em paralelo foram desenvolvidos modelos dos principais dispositivos lineares e não-lineares (e.g. transformador com saturação e compensador estático de reativos) conectados aos sistemas elétricos de potência. $\mathrm{O}$ algoritmo do MICTH juntamente com os modelos de componentes desenvolvidos foram implementados em $\mathrm{C}++$, com programação orientada a objetos e utilizando técnicas de programação esparsa. Os resultados das simulações no domínio da freqüência foram validados a partir de comparações com resultados de simulações no domínio do tempo obtidas com o programa ATP/EMTP (Alternative Transients Program of Electromagnetic Transients Program).

\section{ANÁLISE HARMÔNICA}

Metodologias para análise harmônica podem ser baseadas em formulações matemáticas no domínio do tempo ou no domínio da freqüência.

O cálculo das componentes harmônicas no domínio do tempo consiste no emprego de métodos de integração numérica para obter a solução, no tempo, de um conjunto de equações diferenciais que modelam o comportamento dinâmico dos ele- 
mentos e equipamentos conectados a um sistema de potência. Apesar dos resultados precisos, esta metodologia apresenta um esforço computacional elevado, mesmo para sistemas relativamente pequenos, visto que é necessário simular o sistema durante um longo período até que as tensões e correntes do sistema elétrico atinjam o regime permanente. A partir deste instante pode-se então extrair as componentes harmônicas das tensões e das correntes do sistema utilizando rotinas de Transformada Rápida de Fourier (FFT, Fast Fourier Transform).

Por outro lado, métodos baseados em formulações no domínio da freqüência operam diretamente com os fasores de tensão e de corrente. Estes métodos requerem menor esforço computacional já que o tempo de simulação depende diretamente da quantidade de harmônicos investigados (simulados) e do número de iterações necessárias para a convergência do sistema matricial. Entretanto, os métodos no domínio da freqüência apresentam limitações quanto à representação de cargas não-lineares como dispositivos com chaveamento periódico (conversores estáticos) e de componentes dependentes da freqüência (IEEE, 1996; Arrillaga et alii, 1985).

A metodologia proposta no presente trabalho foi desenvolvida com o intuito de suprir as limitações citadas anteriormente, ou seja, apresentando uma formulação com baixo esforço computacional, porém com boa precisão de resultados.

\section{METODOLOGIA PROPOSTA}

O MICTH realiza o cálculo do fluxo de potência harmônico trifásico baseado nas equações de injeção de correntes empregando o método de solução iterativa de Newton-Raphson. Esta metodologia é uma generalização não trivial para o domínio da frequiência do fluxo de potência convencional baseado no método de injeção de correntes trifásicos conhecido como MICT e descrito por Garcia et alii (2000, 2001a, 2001b, 2004), Variz et alii (2002, 2003) e Da Costa et alii (1997). O MICTH segue as características das formulações no domínio da freqüência, entretanto com uma série de inovações e vantagens permitindo que o sistema elétrico como um todo seja representado de forma mais completa e real. Dentre elas podemos citar (Variz et alii, 2006a, 2006b):

- A representação trifásica dos elementos da rede através de componentes de fase, possibilitando a modelagem e a simulação de sistemas elétricos desequilibrados e com acoplamentos magnéticos entre fases, mantendo sua identidade, uma vez que não é realizado nenhum tipo de transformação ou simplificação;

- A inclusão de acoplamentos harmônicos, permitindo a modelagem de cargas não lineares considerando todas as interações entre freqüências e fontes harmônicas dis- tintas;

- A utilização do sistema matricial baseado nas equações de injeção de corrente, obtendo um baixo índice de atualizações da matriz Jacobiana durante o processo iterativo.

O sistema matricial básico resolvido iterativamente é apresentado em (1). Esta matriz é formada por $H$ submatrizes, ou seja, formada por $H$ subsistemas, com cada uma representando um subsistema matricial em uma determinada freqüência.

$$
\left[\begin{array}{c}
{\left[\Delta I^{a b c}\right]_{0}} \\
{\left[\Delta J^{a b c}\right]_{1}} \\
{\left[\Delta I^{a b c}\right]_{2}} \\
\vdots \\
{\left[\Delta I^{a b c}\right]_{H}}
\end{array}\right]=\left[\begin{array}{ccccc}
{\left[J^{a b c}\right]_{00}} & {\left[J^{a b c}\right]_{01}} & {\left[J^{a b c}\right]_{02}} & \ldots & {\left[J^{a b c}\right]_{0 H}} \\
{\left[J^{a b c}\right]_{10}} & {\left[J^{a b c}\right]_{11}} & {\left[J^{a b c}\right]_{12}} & \ldots & {\left[J^{a b c}\right]_{1 H}} \\
{\left[J^{a b c}\right]_{20}} & {\left[J^{a b c}\right]_{21}} & {\left[J^{a b c}\right]_{22}} & \ldots & {\left[J^{a b c}\right]_{2 H}} \\
\vdots & \vdots & \vdots & \ddots & \vdots \\
{\left[J^{a b c}\right]_{H 0}} & {\left[J^{a b c}\right]_{H 1}} & {\left[J^{a b c}\right]_{H 2}} & \ldots & {\left[J^{a b c}\right]_{H H}}
\end{array}\right]\left[\begin{array}{c}
{\left[\Delta V^{a b c}\right]_{0}} \\
{\left[\Delta V^{a b c}\right]_{1}} \\
{\left[\Delta V^{a b c}\right]_{2}} \\
\vdots \\
{\left[\Delta V^{a b c}\right]_{H}}
\end{array}\right]
$$

onde:

$\left[\Delta I^{a b c}\right]_{h}$ é o vetor trifásico complexo com os resíduos de correntes nodais do subsistema de freqüência $h$;

$\left[J^{a b c}\right]_{h h}$ é a matriz Jacobiana trifásica complexa do subsistema de frequiência $h$;

$\left[J^{a b c}\right]_{h n}$ é a matriz Jacobiana trifásica complexa representando os acoplamentos entre as freqüências $h$ e $n$;

$\left[\Delta V^{a b c}\right]_{h}$ é o vetor trifásico complexo com os incrementos de tensões nodais do subsistema de freqüência $h$.

Estes vetores e matrizes são trifásicos e complexos, formados por componentes de fase divididos em coordenadas reais e imaginárias. Portanto, apresentam dimensões iguais a:

$$
6(K+x)
$$

onde:

$K$ é o número de barras do sistema;

$x$ é a quantidade de equações adicionais de controle. 
Para um sistema elétrico com $K$ barras, o subsistema matricial em uma determinada freqüência é apresentado por (3). Cada bloco possui dimensão 3 , pois são formados pelos componentes das três fases, onde:

$\left[\Delta I_{m_{k}}^{a b c}\right]_{h} \mathbf{e}\left[\Delta I_{r_{k}}^{a b c}\right]_{h}$ são, respectivamente, os vetores trifásicos com as partes imaginária e real dos resíduos de corrente nodal da barra $k$ na freqüência harmônica $h$;

$\left[\frac{\partial \Delta I_{m_{k}}^{a b c}}{\partial V_{r_{j}}^{a b c}}\right]_{h h},\left[\frac{\partial \Delta I_{m_{k}}^{a b c}}{\partial V_{m_{j}}^{a b c}}\right]_{h h},\left[\frac{\partial \Delta I_{r_{k}}^{a b c}}{\partial V_{r_{j}}^{a b c}}\right]_{h h}$ e $\left[\frac{\partial \Delta I_{r_{k}}^{a b c}}{\partial V_{m_{j}}^{a b c}}\right]_{h h}$ são blocos trifásicos da matriz Jacobiana na frequiência harmônica $h$ formados pelas derivadas parciais dos resíduos de corrente nodal na barra $k$ em relação à tensão nodal da barra $j$;

$\left[\Delta V_{r_{j}}^{a b c}\right]_{h} \mathbf{e}\left[\Delta V_{m_{j}}^{a b c}\right]_{h h}$ são os vetores trifásicos compostos, respectivamente, pelas parcelas real e imaginária dos incrementos de tensão nodal na barra $j$ na freqüência harmônica $h$.

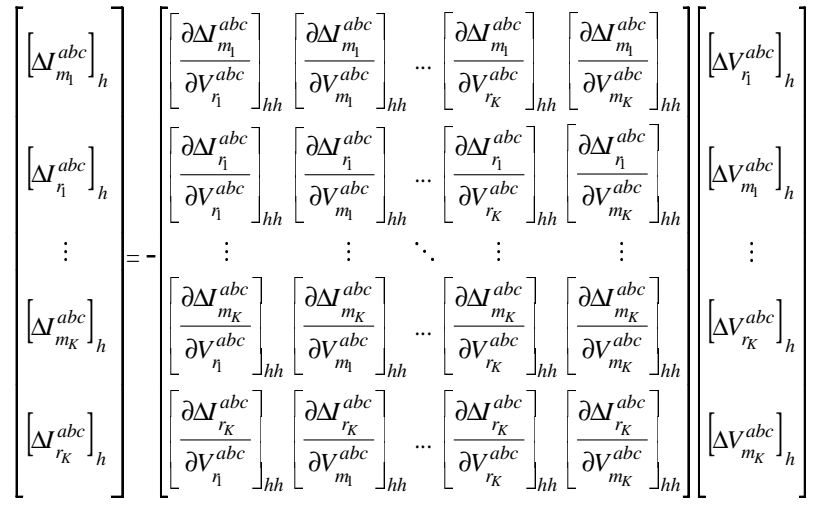

Será mostrado na próxima seção que cada dispositivo apresenta uma parcela de contribuição tanto para o vetor de resíduos como para os elementos da matriz Jacobiana.

O agrupamento de todos os subsistemas matriciais em um único sistema unificado possibilita a incorporação das contribuições dos acoplamentos harmônicos diretamente na solução iterativa. Estas contribuições se encontram nos blocos fora da diagonal principal da matriz Jacobiana. O uso deste recurso permite a implementação de modelos mais completos e precisos dos dispositivos, cuja geração harmônica é dependente de distorções que ocorrem em diversas freqüências. Portanto a dimensão do sistema matricial unificado é dada por: onde $H$ é o número de freqüências harmônicas simuladas.

Assim, devido às elevadas dimensões do sistema matricial resultante, a implementação computacional do MICTH foi realizada através da linguagem $\mathrm{C}++$ com programação orientada a objetos. Além disso, as topologias dos sistemas elétricos de potência e a estrutura de blocos da matriz Jacobiana proporcionaram a aplicação de técnicas de programação esparsa para a otimização do processamento matemático (Tinney, 1967).

\section{MODELAGEM DE DISPOSITIVOS}

A modelagem dos componentes da rede é realizada através de circuitos equivalentes expressos por resistências, capacitâncias e indutâncias próprias e mútuas. Vale ressaltar que, no presente trabalho, a dependência da resistência com a freqüência devido ao efeito pelicular não foi explorada. Essa consideração foi feita porque este fenômeno é mais expressivo em freqüências harmônicas elevadas. No entanto, a metodologia proposta não apresenta nenhuma limitação para a representação desta dependência. A seguir são apresentados os modelos harmônicos dos principais dispositivos de rede operando em regime permanente.

\subsection{Linha de Transmissão}

Sendo o principal meio utilizado para a interconexão dos dispositivos que compõem um sistema elétrico de potência, as linhas de transmissão são caminhos naturais para a propagação de distorções harmônicas pela rede. Portanto, devem ser adequadamente representadas a fim de obter com precisão os fluxos de harmônicas pelo sistema. O circuito equivalente adotado para esta representação é o modelo $\pi$ ilustrado na Figura 1.

Desta forma, as contribuições da LT (linha de transmissão) no sistema matricial (1) nas freqüências harmônicas $h$ e $n$ são apresentadas por:

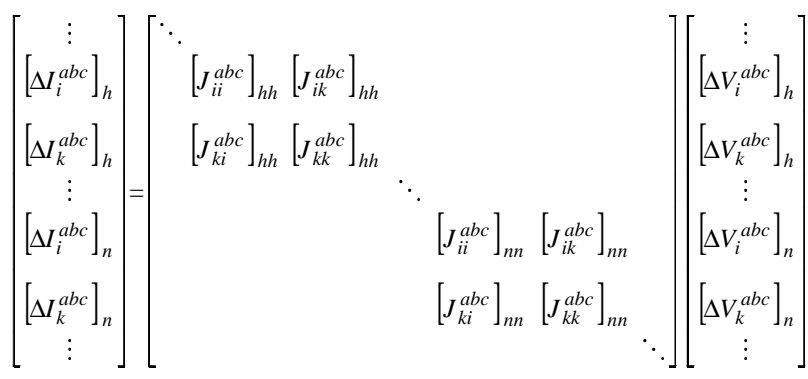

$$
6(H K+x)
$$

onde: 


$$
\begin{gathered}
{\left[\Delta I_{i}^{a b c}\right]_{h}=-\left[I_{i k}^{a b c}\right]_{h}^{L T}=} \\
-\left[Y_{i i}^{a b c}\right]_{h}^{L T}\left[V_{i}^{a b c}\right]_{h}+\left[Y_{i k}^{a b c}\right]_{h}^{L T}\left[V_{k}^{a b c}\right]_{h} \\
{\left[J_{i i}^{a b c}\right]_{h h}=\left[J_{k k}^{a b c}\right]_{h h}=\left[Y_{i i}^{a b c}\right]_{h}^{L T}=\left[Z^{a b c}\right]_{h}^{-1}+\left[Y_{s h}^{a b c}\right]_{h}} \\
{\left[J_{i k}^{a b c}\right]_{h h}=\left[J_{k i}^{a b c}\right]_{h h}=-\left[Y_{i k}^{a b c}\right]_{h}^{L T}=-\left[Z^{a b c}\right]_{h}^{-1}}
\end{gathered}
$$

$\left[\Delta I_{i}^{a b c}\right]_{h}$ é a contribuição da LT no vetor de resíduos de corrente da barra $i$ na freqüência $h$;

$\left[J_{i k}^{a b c}\right]_{h h}$ é a contribuição da LT na matriz Jacobiana no bloco $i k$ na freqüência $h$;

$\left[I_{i k}^{a b c}\right]_{h}^{L T}$ é o vetor trifásico e complexo do fluxo de corrente na LT entre os terminais $i$ e $k$ na freqüência $h$;

$\left[Y_{i k}^{a b c}\right]_{h}^{L T}$ é o bloco $i k$ da matriz admitância nodal trifásico e complexo da LT na freqüência $h$;

$\left[Z^{a b c}\right]_{h}$ é o bloco de impedância trifásico e complexo formado pelos elementos série da LT na frequiência $h$;

$\left[Y_{s h}^{a b c}\right]_{h}$ é o bloco de admitância complexo trifásico formado pelos elementos em derivação da LT na freqüência $h$.

Note que as contribuições são dadas em função da matriz de admitância nodal do componente, portanto, as atualizações na matriz Jacobiana são constantes durante todo o processo iterativo.

\subsection{Carga}

As cargas em um sistema são responsáveis não somente pela variação de tensão da rede, mas também podem afetar as

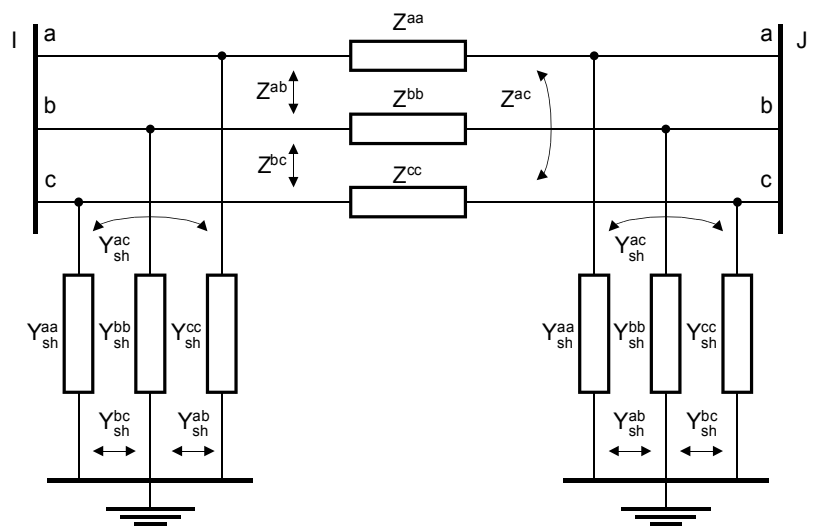

Figura 1: Circuito $\pi$ com parâmetros concentrados de uma linha de transmissão trifásica. condições de ressonância e de fluxo harmônico no sistema. A modelagem de carga não é simples, pois sua composição é difícil de ser estimada já que depende de fatores como tempo, clima e economia.

Neste trabalho o modelo proposto para carga é dividido em duas partes, um para a frequiência fundamental e outro para as demais freqüências. Para a freqüência fundamental, a carga é modelada em função da potência consumida dependente da tensão. Portanto, para uma carga conectada na barra $k$, os fluxos de corrente entre os ramos de fase $s$ e $t$ separados em componentes imaginária e real são dados, respectivamente, por:

$$
\begin{aligned}
I_{L_{m_{k}}}^{s t} & =\frac{P_{L_{k}}^{s t} V_{m_{k}}^{s t}-Q_{L_{k}}^{s t} V_{r_{k}}^{s t}}{\left(V_{k}^{s t}\right)^{2}} \\
I_{L_{r_{k}}}^{s t} & =\frac{P_{L_{k}}^{s t} V_{r_{k}}^{s t}+Q_{L_{k}}^{s t} V_{m_{k}}^{s t}}{\left(V_{k}^{s t}\right)^{2}}
\end{aligned}
$$

onde:

o índice $s t$ representa os ramos entre as fases $a b, b c$ e $c a$ para cargas conectadas em delta, e os ramos entre as fases $(a, b$ e $c)$ e o neutro (o) para cargas em estrela;

$P_{L_{k}}^{s t}$ e $Q_{L_{k}}^{s t}$ são as potências ativa e reativa consumidas pela carga e expressas por uma representação polinomial (ZIP) conforme mostrado a seguir:

$$
\begin{gathered}
P_{L_{k}}^{s t}=P_{0_{k}}^{s t}\left[p_{Z}{\overline{V_{k}^{s t}}}^{2}+p_{I} \overline{V_{k}^{s t}}+p_{P}\right]= \\
P_{Z_{k}}^{s t}{\overline{V_{k}^{s t}}}^{2}+P_{I_{k}}^{s t} \overline{V_{k}^{s t}}+P_{P_{k}}^{s t} \\
Q_{L_{k}}^{s t}=Q_{0_{k}}^{s t}\left[q_{Z}{\overline{V_{k}^{s t}}}^{2}+q_{I} \overline{V_{k}^{s t}}+q_{P}\right]= \\
Q_{Z_{k}}^{s t}{\overline{V_{k}^{s t}}}^{2}+Q_{I_{k}}^{s t} \overline{V_{k}^{s t}}+Q_{P_{k}}^{s t} \\
\overline{V_{k}^{s t}}=\frac{V_{k}^{s t}}{V_{0_{k}}^{s t}} \\
p_{Z}+p_{I}+p_{P}=1 \mathrm{e} q_{Z}+q_{I}+q_{P}=1
\end{gathered}
$$

onde: $P_{0_{k}}^{s t}, Q_{0_{k}}^{s t}$ e $V_{0_{k}}^{s t}$ são as potências e tensão iniciais de operação, respectivamente;

Os termos $p_{Z}, p_{I}, p_{P}, q_{Z}, q_{I}$ e $q_{P}$ em (14) são as parcelas ativa e reativa de impedância, corrente e potência constantes que compõem a carga, respectivamente. 
A representação da carga nas freqüências diferentes da fundamental é realizada pela associação de componentes passivos (ramos $R, L$ e $C$ ) em série ou em paralelo, cujos valores são calculados a partir das potências dadas em (11) e (12) e respeitando o tipo de conexão do componente no barramento.

Portanto, para uma carga conectada em uma barra $k$ do sistema, as contribuições matriciais em (1) nas freqüências fundamental, $h$ e $n$ são apresentadas por:



As contribuições no vetor de resíduos na fase s e na frequiência fundamental para cargas conectadas em estrela são calculadas pelas equações:

$$
\begin{gathered}
\Delta I_{m_{k}}^{s}=-I_{L m_{k}}^{s}=-I_{L m_{k}}^{s o} \\
\Delta I_{r_{k}}^{s}=-I_{L r_{k}}^{s}=-I_{L r_{k}}^{s o}
\end{gathered}
$$

E para cargas em delta são calculadas por:

$$
\begin{gathered}
\Delta I_{m_{k}}^{s}=-I_{L m_{k}}^{s}=-\left(I_{L m_{k}}^{s t}-I_{L m_{k}}^{u s}\right) \\
\Delta I_{r_{k}}^{s}=-I_{L r_{k}}^{s}=-\left(I_{L r_{k}}^{s t}-I_{L r_{k}}^{u s}\right)
\end{gathered}
$$

onde: $I_{L m_{k}}^{s}$ e $I_{L r_{k}}^{s}$ são as correntes nodais consumidas pela carga na fase $s$ separadas em partes imaginária e real.

As contribuições na matriz Jacobiana na freqüência fundamental para cargas conectadas em estrela são realizadas pela seguinte derivada parcial complexa:

$$
\frac{\partial I_{L}^{s}}{\partial V^{s}}=\frac{\partial I_{L}^{s o}}{\partial V^{s o}}
$$

Enquanto que para cargas em delta, ao se desenvolver a derivada aplicando a regra da cadeia, tem-se:

$$
\frac{\partial I_{L}^{s}}{\partial V^{s}}=\frac{\partial I_{L}^{s t}}{\partial V^{s}}-\frac{\partial I_{L}^{u s}}{\partial V^{s}}=\frac{\partial I_{L}^{s t}}{\partial V^{s t}}+\frac{\partial I_{L}^{u s}}{\partial V^{u s}}
$$

$$
\begin{gathered}
\frac{\partial I_{L}^{s}}{\partial V^{t}}=\frac{\partial I_{L}^{s t}}{\partial V^{t}}-\frac{\partial I_{L}^{u s}}{\partial V^{t}}=\frac{\partial I_{L}^{s t}}{\partial V^{t}}-0=-\frac{\partial I_{L}^{s t}}{\partial V^{s t}} \\
\frac{\partial I_{L}^{s}}{\partial V^{u}}=\frac{\partial I_{L}^{s t}}{\partial V^{u}}-\frac{\partial I_{L}^{u s}}{\partial V^{u}}=0-\frac{\partial I_{L}^{u s}}{\partial V^{u}}=-\frac{\partial I_{L}^{u s}}{\partial V^{u s}}
\end{gathered}
$$

onde as expressões das derivadas parciais complexas dadas em (20) a (23) são calculadas por:

$$
\begin{gathered}
\frac{\partial I_{L m_{k}}^{s t}}{\partial V_{r_{k}}^{s t}}=-\frac{Q_{P_{k}}^{s t}\left[\left(V_{m_{k}}^{s t}\right)^{2}-\left(V_{r_{k}}^{s t}\right)^{2}\right]+2 P_{P_{k}}^{s t} V_{m_{k}}^{s t} V_{r_{k}}^{s t}}{\left(V_{k}^{s t}\right)^{4}}- \\
\frac{P_{I_{k}}^{s t} V_{m_{k}}^{s t} V_{r_{k}}^{s t}+Q_{I_{k}}^{s t}\left(V_{m_{k}}^{s t}\right)^{2}}{\left(V_{k}^{s t}\right)^{3} V_{0_{k}}^{s t}}-\frac{Q_{Z_{k}}^{s t}}{\left(V_{0_{k}}^{s t}\right)^{2}} \\
\frac{\partial I_{L m_{k}}^{s t}}{\partial V_{m_{k}}^{s t}}=-\frac{P_{P_{k}}^{s t}\left[\left(V_{m_{k}}^{s t}\right)^{2}-\left(V_{r_{k}}^{s t}\right)^{2}\right]-2 Q_{P_{k}}^{s t} V_{m_{k}}^{s t} V_{r_{k}}^{s t}}{\left(V_{k}^{s t}\right)^{4}}+ \\
\frac{Q_{I_{k}}^{s t} V_{m_{k}}^{s t} V_{r_{k}}^{s t}+P_{I_{k}}^{s t}\left(V_{r_{k}}^{s t}\right)^{2}}{\left(V_{k}^{s t}\right)^{3} V_{0_{k}}^{s t}}-\frac{P_{Z_{k}}^{s t}}{\left(V_{0_{k}}^{s t}\right)^{2}}
\end{gathered}
$$

$$
\begin{aligned}
\frac{\partial I_{L r_{k}}^{s t}}{\partial V_{r_{k}}^{s t}}=\frac{P_{P_{k}}^{s t}\left[\left(V_{m_{k}}^{s t}\right)^{2}-\left(V_{r_{k}}^{s t}\right)^{2}\right]-2 Q_{P_{k}}^{s t} V_{m_{k}}^{s t} V_{r_{k}}^{s t}}{\left(V_{k}^{s t}\right)^{4}}- \\
\frac{Q_{I_{k}}^{s t} V_{m_{k}}^{s t} V_{r_{k}}^{s t}-P_{I_{k}}^{s t}\left(V_{m_{k}}^{s t}\right)^{2}}{\left(V_{k}^{s t}\right)^{3} V_{0_{k}}^{s t}}+\frac{P_{Z_{k}}^{s t}}{\left(V_{0_{k}}^{s t}\right)^{2}}
\end{aligned}
$$

$$
\begin{gathered}
\frac{\partial I_{L r_{k}}^{s t}}{\partial V_{m_{k}}^{s t}}=-\frac{Q_{P_{k}}^{s t}\left[\left(V_{m_{k}}^{s t}\right)^{2}-\left(V_{r_{k}}^{s t}\right)^{2}\right]+2 P_{P_{k}}^{s t} V_{m_{k}}^{s t} V_{r_{k}}^{s t}}{\left(V_{k}^{s t}\right)^{4}}- \\
\frac{P_{I_{k}}^{s t} V_{m_{k}}^{s t} V_{r_{k}}^{s t}-Q_{I_{k}}^{s t}\left(V_{r_{k}}^{s t}\right)^{2}}{\left(V_{k}^{s t}\right)^{3} V_{0_{k}}^{s t}}+\frac{Q_{Z_{k}}^{s t}}{\left(V_{0_{k}}^{s t}\right)^{2}}
\end{gathered}
$$

Para as demais freqüências, as contribuições no vetor de resíduos de corrente e na matriz Jacobiana são calculadas a partir da matriz admitância nodal formada pela associação dos componentes passivos obtidos através das seguintes etapas:

- Cálculo da impedância de carga equivalente na freqüência fundamental através da equação:

$$
Z_{k}^{s t}=\frac{\left(V_{k}^{s t}\right)^{2}\left(P_{L_{k}}^{s t}+j Q_{L_{k}}^{s t}\right)}{\left(P_{L_{k}}^{s t}\right)^{2}+\left(Q_{L_{k}}^{s t}\right)^{2}}
$$

onde as potências de carga são calculadas em (11) e (12);

- Obtenção dos componentes passivos formados pela resistência, indutância e capacitância calculadas a partir da impedância equivalente na freqüência fundamental (28); 
- Construção da matriz admitância nodal para as freqüências diferentes da fundamental formada pelos componentes passivos obtidos na etapa anterior.

Portanto para as freqüências diferentes da fundamental temse:

$$
\begin{gathered}
{\left[\Delta I_{k}^{a b c}\right]_{h}=-\left[Y_{k k}^{a b c}\right]_{h}^{c a r}\left[V_{k}^{a b c}\right]_{h}} \\
{\left[J_{k k}^{a b c}\right]_{h h}=\left[Y_{k k}^{a b c}\right]_{h}^{c a r}}
\end{gathered}
$$

onde:

$\left[Y_{k k}^{a b c}\right]_{h}^{c a r}$ é a matriz admitância nodal trifásica e complexa da carga na freqüência $h$, calculada a partir da expressão (28);

$\left[V_{k}^{a b c}\right]_{h}$ é o vetor de tensão nodal trifásico e complexo na freqüência $h$.

Note que para cargas do tipo impedância constante, as atualizações na matriz Jacobiana são constantes durante todo o processo iterativo, visto que a matriz admitância nodal permanece inalterada.

\subsection{Gerador}

As unidades geradoras com controle de tensão são modelas através de fontes de tensão senoidal atrás de uma impedância. A característica do modelo desenvolvido é o de realizar injeções de potência ativa conhecidas mantendo-se a tensão nos terminais constantes em um valor pré-definido. O controle desta tensão terminal é realizado através da injeção ou absorção de reativos (Garcia et alii, 2004; Variz et alii, 2003).

As contribuições no sistema matricial unificado de um gerador conectado na barra $k$ são apresentadas por:

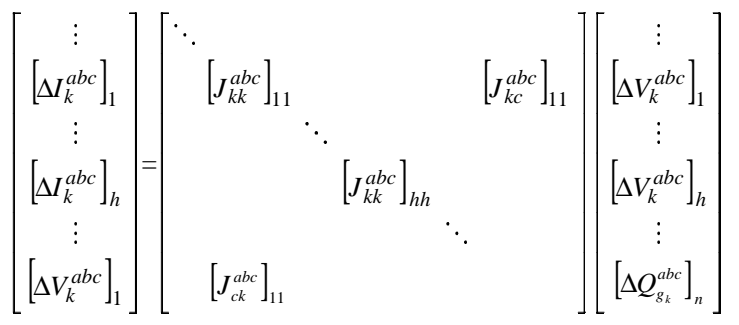

As contribuições na fase $s$ do vetor de resíduos na freqüência fundamental em (31) são dadas por:

$$
\Delta I_{m_{k}}^{s}=I_{g m_{k}}^{s}=\frac{P_{g_{k}}^{s} V_{m_{k}}^{s}-Q_{g_{k}}^{s} V_{r_{k}}^{s}}{\left(V_{k}^{s}\right)^{2}}
$$

$$
\begin{aligned}
& \Delta I_{r_{k}}^{s}=I_{g r_{k}}^{s}=\frac{P_{g_{k}}^{s} V_{r_{k}}^{s}+Q_{g_{k}}^{s} V_{m_{k}}^{s}}{\left(V_{k}^{s}\right)^{2}} \\
& \Delta V_{k}^{s}=V_{k_{s p}}^{s}-\sqrt{\left(V_{r_{k}}^{s}\right)^{2}+\left(V_{m_{k}}^{s}\right)^{2}}
\end{aligned}
$$

onde:

$I_{g m_{k}}^{s}$ e $I_{g r_{k}}^{s}$ são as correntes nodais injetadas pelo gerador na fase $s$ separadas em partes imaginária e real;

$P_{g_{k}}^{s}$ e $Q_{g_{k}}^{s}$ são as potências ativa e reativa na fase sinjetadas pelo gerador da barra $k$;

$V_{k_{s p}}^{s}$ é o valor do módulo da tensão especificado na fase $s$ no barramento $k$.

A expressão (34) é responsável pelo controle de tensão através da injeção de reativos. Vale ressaltar que a inclusão desta equação extra no sistema matricial implica na adição de uma nova variável de estado $\Delta Q_{k}^{a b c}$ e conseqüentemente no aumento do sistema matricial unificado, conforme observado em (31).

As contribuições na matriz Jacobiana na frequiência fundamental são realizadas por:

$$
\begin{aligned}
\frac{\partial I_{g m_{k}}^{s}}{\partial V_{r_{k}}^{s}}= & \frac{\partial I_{g r_{k}}^{s}}{\partial V_{m_{k}}^{s}}= \\
& \frac{Q_{g_{k}}^{s}\left[\left(V_{r_{k}}^{s}\right)^{2}-\left(V_{m_{k}}^{s}\right)^{2}\right]-2 P_{g_{k}}^{s} V_{m_{k}}^{s} V_{r_{k}}^{s}}{\left(V_{k}^{s}\right)^{4}}
\end{aligned}
$$

$$
\begin{gathered}
\frac{\partial I_{g m_{k}}^{s}}{\partial V_{m_{k}}^{s}}=-\frac{\partial I_{g r_{k}}^{s}}{\partial V_{r_{k}}^{s}}= \\
\frac{P_{g_{k}}^{s}\left[\left(V_{r_{k}}^{s}\right)^{2}-\left(V_{m_{k}}^{s}\right)^{2}\right]+2 Q_{g_{k}}^{s} V_{m_{k}}^{s} V_{r_{k}}^{s}}{\left(V_{k}^{s}\right)^{4}} \\
\frac{\partial I_{g m_{k}}^{s}}{\partial Q_{g_{k}}^{s}}=\frac{V_{r_{k}}^{s}}{\left(V_{r_{k}}^{s}\right)^{2}+\left(V_{m_{k}}^{s}\right)^{2}} \\
\frac{\partial I_{g r_{k}}^{s}}{\partial Q_{g_{k}}^{s}}=-\frac{V_{m_{k}}^{s}}{\left(V_{r_{k}}^{s}\right)^{2}+\left(V_{m_{k}}^{s}\right)^{2}} \\
\frac{\partial \Delta V_{k}^{s}}{\partial V_{r_{k}}^{s}}=-\frac{V_{r_{k}}^{s}}{\sqrt{\left(V_{r_{k}}^{s}\right)^{2}+\left(V_{m_{k}}^{s}\right)^{2}}} \\
\frac{\partial \Delta V_{k}^{s}}{\partial V_{m_{k}}^{s}}=-\frac{V_{m_{k}}^{s}}{\sqrt{\left(V_{r_{k}}^{s}\right)^{2}+\left(V_{m_{k}}^{s}\right)^{2}}}
\end{gathered}
$$

Sendo que as derivadas parciais (37) a (40) são incluídas no subsistema matricial em conseqüência da adição da equação extra (34). 


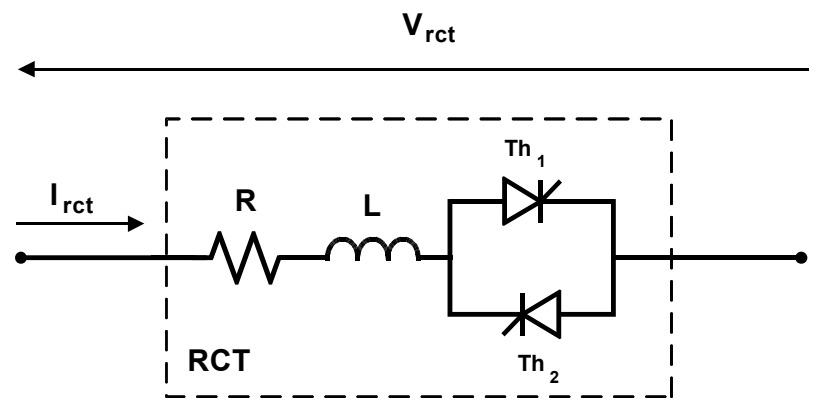

Figura 2: Circuito equivalente do Reator Controlado a Tiristor.

Para as frequiências diferentes da fundamental, as contribuições matriciais são realizadas por (29) e (30), porém com a matriz admitância nodal calculada pela impedância equivalente do gerador.

Note que o modelo incorpora o controle de tensão dentro do sistema matricial, permitindo inclusive o controle em barras remotas.

\subsection{Reator Controlado a Tiristor}

Conversores eletrônicos de potência são fontes de harmônicos cuja utilização em sistemas elétricos tem aumentado consideravelmente nos últimos anos. Modelos precisos destes equipamentos tem sido tema constante de pesquisas. Esses dispositivos são difíceis de serem modelados, pois suas características não-lineares não são facilmente representadas por modelos harmônicos equivalentes. Geralmente a análise em regime permanente destes dispositivos é baseada em suas características no domínio do tempo (Arrillaga et alii, 1997). Porém, como as simulações no domínio do tempo são lentas, o uso de técnicas de simulação no domínio da frequiência em conjunto com algoritmos para solução de equações nãolineares constituem um ferramenta interessante e rápida para avaliar o impacto desses dispositivos nos SEP (Semlyen et alii, 1988; Xu et alii, 1991; Acha e Madrigal, 2001).

A Figura 2 mostra um reator controlado a tiristores (RCT). Ele é um compensador eletrônico de potência simples que pode ser facilmente utilizado como referência na modelagem de dispositivos mais sofisticados como o Compensador Estático de Reativo (CER) e o Compensador Série Controlado a Tiristores (CSCT). O modelo apresentado a seguir é definido como uma injeção de corrente com tensão controlada no domínio da freqüência, sendo os componentes desta corrente expressos analiticamente em função da tensão, permitindo desta forma uma linearização direta (Lima et alii, 2003).

A Figura 3 ilustra a característica de tensão e corrente nos terminais de um RCT. O regime de condução descontínua da



Figura 3: Características no domínio do tempo do RCT.

corrente é definido pelos instantes de disparo $\left(t_{o n 1}\right.$ e $\left.t_{o n 2}\right)$ e de corte $\left(t_{o f f 1}\right.$ e $\left.t_{o f f 2}\right)$ dos tiristores $\mathrm{Th}_{1}$ e $\mathrm{Th}_{2}$, respectivamente.

Durante o período de condução a seguinte equação diferencial ordinária (EDO) é válida:

$$
L \frac{d i(t)}{d t}+R i(t)=v(t)
$$

Assumindo a tensão distorcida e expressa por uma série de Fourier dada por:

$$
v(t)=V_{0}+\sum_{n=1}^{\infty}\left(V_{r_{n}} \cos (n \omega t)+V_{m_{n}} \operatorname{sen}(n \omega t)\right)
$$

onde:

$V_{r_{n}}$ e $V_{m_{n}}$ são as componentes real e imaginária da tensão de ordem harmônica $n$;

$V_{0}$ é a componente $\mathrm{CC}$ da tensão;

$\omega$ é a freqüência angular fundamental.

Substituindo (42) em (41) tem-se que a corrente pelo RCT é calculada por:

$$
\begin{aligned}
& i(t)=K e^{-\frac{R}{L}\left(t-t_{o n}\right)}+\frac{V_{0}}{R}+ \\
& \sum_{n=1}^{\infty}\left(\frac{V_{r_{n}}}{Z_{n}} \cos \left(n \omega t-\varphi_{n}\right)+\frac{V_{m_{n}}}{Z_{n}} \operatorname{sen}\left(n \omega t-\varphi_{n}\right)\right)
\end{aligned}
$$

onde:

$$
Z_{n}=\sqrt{R^{2}+(n \omega L)^{2}}
$$




$$
\begin{gathered}
\varphi_{n}=\tan ^{-1}\left(\frac{n \omega L}{R}\right) \\
K=-\frac{V_{0}}{R}- \\
\sum_{n=1}^{\infty}\left(\frac{V_{r_{n}}}{Z_{n}} \cos \left(n \omega t_{\text {on }}-\varphi_{n}\right)+\frac{V_{m_{n}}}{Z_{n}} \operatorname{sen}\left(n \omega t_{\text {on }}-\varphi_{n}\right)\right)
\end{gathered}
$$

Expandindo (43) através de sua série de Fourier (Lathi, 2005), considerando os períodos de condução definidos pelos instantes $t_{o n j}$ e $t_{o f f j}$, tem-se que as componentes real e imaginária dos harmônicos da corrente pelo RCT, na freqüência $h$, para o ramo entre as fases $s$ e $t$ são dados por:

$$
\begin{gathered}
I_{r c t r_{h}}^{s t}=\frac{\omega}{\pi} \sum_{j=1}^{2}\left[I^{\prime}\left(h, t_{o f f_{j}}\right)-I^{\prime}\left(h, t_{o n_{j}}\right)\right] \\
I_{r c t m_{h}}^{s t}=\frac{\omega}{\pi} \sum_{j=1}^{2}\left[I^{\prime \prime}\left(h, t_{o f f_{j}}\right)-I^{\prime \prime}\left(h, t_{o n_{j}}\right)\right]
\end{gathered}
$$

onde as funções auxiliares $I^{\prime}\left(h, t_{j}\right)$ e $I^{\prime \prime}\left(h, t_{j}\right)$ são calculadas, respectivamente, como se segue:

$$
\begin{gathered}
I^{\prime}\left(h, t_{j}\right)=\left[\frac{V_{0}^{s t} \operatorname{sen}\left(h \omega t_{j}\right)}{h \omega R}\right]+ \\
\sum_{n=1}^{\infty}\left[\frac { V _ { r _ { n } } ^ { s t } } { 2 \omega Z _ { n } } \left[\frac{\operatorname{sen}\left((h+n) \omega t_{j}-\varphi_{n}\right)}{h+n}+\right.\right. \\
n \neq h \\
\left.\left.\quad \frac{\operatorname{sen}\left((h-n) \omega t_{j}-\varphi_{n}\right)}{h-n}\right]\right]+ \\
{\left[\begin{array}{c}
V_{r_{h}}^{s t} \\
2 \omega Z_{h}
\end{array}\left[\omega t_{j} \cos \left(\varphi_{h}\right)+\frac{\operatorname{sen}\left(2 h \omega t_{j}-\varphi_{h}\right)}{2 h}\right]\right]+} \\
\sum_{\substack{n=1 \\
n \neq h}}^{\infty}\left[-\frac{V_{m_{n}}^{s t}}{2 \omega Z_{n}}\left[\frac{\cos \left((n-h) \omega t_{j}-\varphi_{n}\right)}{n-h}+\right.\right. \\
\left.\frac{\cos \left((n+h) \omega t_{j}-\varphi_{n}\right)}{n+h}\right]+ \\
{\left[\frac{K e^{-\frac{R}{L}\left(t_{j}-t_{o n_{j}}\right)} L}{\left(Z_{h}\right)^{2}}\left[-R \cos \left(h \omega t_{j}\right)+h \omega L \operatorname{sen}\left(h \omega t_{j}\right)\right]\right]}
\end{gathered}
$$

$$
\begin{aligned}
& I^{\prime \prime}\left(h, t_{j}\right)=\left[-\frac{V_{0}^{s t} \cos \left(h \omega t_{j}\right)}{h \omega R}\right]+ \\
& \sum_{n=1}^{\infty}\left[-\frac{V_{r_{n}}^{s t}}{2 \omega Z_{n}}\left[\frac{\cos \left((h+n) \omega t_{j}-\varphi_{n}\right)}{h+n}+\right.\right. \\
& n \neq h \\
& \left.\left.\frac{\cos \left((h-n) \omega t_{j}-\varphi_{n}\right)}{h-n}\right]\right]+ \\
& {\left[\frac{V_{r_{h}}^{s t}}{2 \omega Z_{h}}\left[\omega t_{j} \operatorname{sen}\left(\varphi_{h}\right)-\frac{\cos \left(2 h \omega t_{j}-\varphi_{h}\right)}{2 h}\right]\right]+} \\
& \sum_{n=1}^{\infty}\left[\frac { V _ { m _ { n } } ^ { s t } } { 2 \omega Z _ { n } } \left[\frac{\operatorname{sen}\left((n-h) \omega t_{j}-\varphi_{n}\right)}{n-h}-\right.\right. \\
& n \neq h \\
& \left.\left.\frac{\operatorname{sen}\left((n+h) \omega t_{j}-\varphi_{n}\right)}{n+h}\right]\right]+ \\
& {\left[\frac{V_{m_{h}}^{s t}}{2 \omega Z_{h}}\left[\omega t_{j} \cos \left(\varphi_{h}\right)-\frac{\operatorname{sen}\left(2 h \omega t_{j}-\varphi_{h}\right)}{2 h}\right]\right]+} \\
& {\left[\frac{K e^{-\frac{R}{L}\left(t_{j}-t_{o n_{j}}\right)} L}{\left(Z_{h}\right)^{2}}\left[-R \operatorname{sen}\left(h \omega t_{j}\right)-h \omega L \cos \left(h \omega t_{j}\right)\right]\right]}
\end{aligned}
$$

As contribuições matriciais nas freqüências fundamental, $h \mathrm{e}$ $n$ de um RCT conectado na barra $k$ são dadas por:

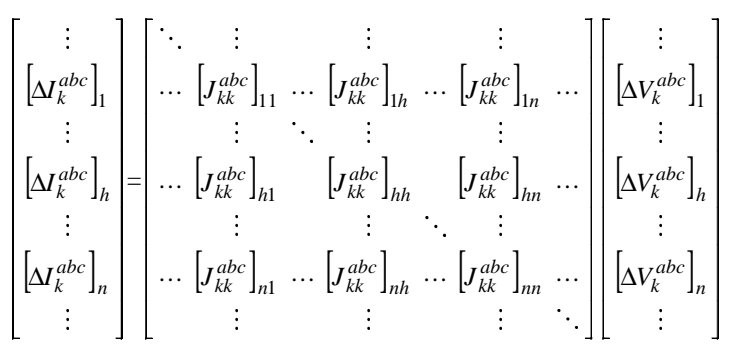

Com o RCT conectado em estrela, as contribuições no vetor de resíduos na fase $s$ são obtidas por:

$$
\begin{aligned}
\Delta I_{m_{k}}^{s} & =-I_{r c t m_{k}}^{s}=-I_{r c t m_{k}}^{s o} \\
\Delta I_{r_{k}}^{s} & =-I_{r c t r_{k}}^{s}=-I_{r c t r_{k}}^{s o}
\end{aligned}
$$

Enquanto que para o RCT em delta são calculadas por:

$$
\Delta I_{m_{k}}^{s}=-I_{r c t m_{k}}^{s}=-\left(I_{r c t m_{k}}^{s t}-I_{r c t m_{k}}^{u s}\right)
$$




$$
\Delta I_{r_{k}}^{s}=-I_{r c t r_{k}}^{s}=-\left(I_{r c t r_{k}}^{s t}-I_{r c t r_{k}}^{u s}\right)
$$

As contribuições na matriz Jacobiana conforme o tipo de conexão do RCT são análogas às apresentadas em (20) a (23), porém com as derivadas parciais calculadas conforme mostrado a seguir:

$$
\begin{gathered}
\frac{\partial I_{r c t r_{h}}^{s}}{\partial V_{r_{n}}^{s}}=\frac{\omega}{\pi} \sum_{j=1}^{2}\left[\frac{\partial I^{\prime}\left(h, t_{o f f_{j}}\right)}{\partial V_{r_{n}}}-\frac{\partial I^{\prime}\left(h, t_{o n_{j}}\right)}{\partial V_{r_{n}}}\right] \\
\frac{\partial I_{r c t r_{h}}^{s}}{\partial V_{m_{n}}^{s}}=\frac{\omega}{\pi} \sum_{j=1}^{2}\left[\frac{\partial I^{\prime}\left(h, t_{o f f_{j}}\right)}{\partial V_{m_{n}}}-\frac{\partial I^{\prime}\left(h, t_{o n_{j}}\right)}{\partial V_{m_{n}}}\right] \\
\frac{\partial I_{r c t m_{h}}^{s}}{\partial V_{r_{n}}^{s}}=\frac{\omega}{\pi} \sum_{j=1}^{2}\left[\frac{\partial I^{\prime \prime}\left(h, t_{o f f_{j}}\right)}{\partial V_{r_{n}}}-\frac{\partial I^{\prime \prime}\left(h, t_{o n_{j}}\right)}{\partial V_{r_{n}}}\right] \\
\frac{\partial I_{r c t m_{h}}^{s}}{\partial V_{m_{n}}^{s}}=\frac{\omega}{\pi} \sum_{j=1}^{2}\left[\frac{\partial I^{\prime \prime}\left(h, t_{o f f_{j}}\right)}{\partial V_{m_{n}}}-\frac{\partial I^{\prime \prime}\left(h, t_{o n_{j}}\right)}{\partial V_{m_{n}}}\right]
\end{gathered}
$$

Para os blocos da diagonal principal da matriz Jacobiana tem-se que as derivadas parciais em (56) a (59) são calculadas por:

$$
\begin{aligned}
& \frac{\partial I^{\prime}\left(h, t_{j}\right)}{\partial V_{r_{h}}}= \\
& \frac{1}{2 \omega Z_{h}}\left[\omega t_{j} \cos \left(\varphi_{h}\right)+\frac{\operatorname{sen}\left(2 h \omega t_{j}-\varphi_{h}\right)}{2 h}-\right. \\
& 2 \omega \cos \left(h \omega t_{o n_{j}}-\varphi_{h}\right) \frac{e^{-\frac{R}{L}\left(t_{j}-t_{o n_{j}}\right)} L}{\left(Z_{h}\right)^{2}} \\
&\left.\quad\left(-R \cos \left(h \omega t_{j}\right)+h \omega L \operatorname{sen}\left(h \omega t_{j}\right)\right)\right]
\end{aligned}
$$

$$
\begin{aligned}
& \frac{\partial I^{\prime}\left(h, t_{j}\right)}{\partial V_{m_{h}}}= \\
& \frac{1}{2 \omega Z_{h}}\left[-\omega t_{j} \operatorname{sen}\left(\varphi_{h}\right)+\frac{\cos \left(2 h \omega t_{j}-\varphi_{h}\right)}{2 h}-\right. \\
& 2 \omega \operatorname{sen}\left(h \omega t_{o n_{j}}-\varphi_{h}\right) \frac{e^{-\frac{R}{L}\left(t_{j}-t_{o n_{j}}\right)} L}{\left(Z_{h}\right)^{2}} \\
& \left.\quad\left(-R \cos \left(h \omega t_{j}\right)+h \omega L \operatorname{sen}\left(h \omega t_{j}\right)\right)\right]
\end{aligned}
$$

$$
\begin{aligned}
& \frac{\partial I "\left(h, t_{j}\right)}{\partial V_{r_{h}}}= \\
& \frac{1}{2 h \omega Z_{h}}\left[h \omega t_{j} \operatorname{sen}\left(\varphi_{h}\right)-\frac{\cos \left(2 h \omega t_{j}-\varphi_{h}\right)}{2}-\right. \\
& 2 \omega \cos \left(h \omega t_{o n_{j}}-\varphi_{h}\right) \frac{e^{-\frac{R}{L}\left(t_{j}-t_{o n_{j}}\right)} L}{\left(Z_{h}\right)^{2}} \\
&\left.\quad\left(-R \operatorname{sen}\left(h \omega t_{j}\right)-h \omega L \cos \left(h \omega t_{j}\right)\right)\right]
\end{aligned}
$$

$$
\begin{aligned}
& \frac{\partial I^{\prime \prime}\left(h, t_{j}\right)}{\partial V_{m_{h}}}= \\
& \frac{1}{2 h \omega Z_{h}}\left[h \omega t_{j} \cos \left(\varphi_{h}\right)-\frac{\operatorname{sen}\left(2 h \omega t_{j}-\varphi_{h}\right)}{2}-\right. \\
& 2 \omega \operatorname{sen}\left(h \omega t_{o n_{j}}-\varphi_{h}\right) \frac{e^{-\frac{R}{L}\left(t_{j}-t_{o n_{j}}\right)} L}{\left(Z_{h}\right)^{2}} \\
&\left.\left(-R \operatorname{sen}\left(h \omega t_{j}\right)-h \omega L \cos \left(h \omega t_{j}\right)\right)\right]
\end{aligned}
$$

Enquanto que os blocos responsáveis pelos acoplamentos harmônicos, situados fora da diagonal principal da matriz Jacobiana, as contribuições (56) a (59) são calculadas a partir das derivadas parciais da corrente harmônica $h$ em relação às tensões harmônicas $n$ e dadas por:

$$
\begin{gathered}
\frac{\partial I^{\prime}\left(h, t_{j}\right)}{\partial V_{r_{n}}}= \\
\frac{-1}{2 \omega Z_{n}}\left[\frac{\operatorname{sen}\left((h+n) \omega t_{j}-\varphi_{n}\right)}{h+n}+\frac{\operatorname{sen}\left((h-n) \omega t_{j}-\varphi_{n}\right)}{h-n}-\right. \\
2 \omega \cos \left(n \omega t_{o n_{j}}-\varphi_{n}\right) \frac{e^{-\frac{R}{L}\left(t_{j}-t_{o n_{j}}\right)} L}{\left(Z_{h}\right)^{2}} \\
\left.\left(-R \cos \left(h \omega t_{j}\right)+h \omega L \operatorname{sen}\left(h \omega t_{j}\right)\right)\right]
\end{gathered}
$$

$$
\begin{gathered}
\frac{\partial I^{\prime}\left(h, t_{j}\right)}{\partial V_{m_{n}}}= \\
\frac{1}{2 \omega Z_{n}}\left[-\frac{\cos \left((n-h) \omega t_{j}-\varphi_{n}\right)}{n-h}+\frac{\cos \left((n+h) \omega t_{j}-\varphi_{n}\right)}{n+h}-\right. \\
2 \omega \operatorname{sen}\left(n \omega t_{o n_{j}}-\varphi_{n}\right) \frac{e^{-\frac{R}{L}\left(t_{j}-t_{o n_{j}}\right)} L}{\left(Z_{h}\right)^{2}} \\
\left.\left(-R \cos \left(h \omega t_{j}\right)+h \omega L \operatorname{sen}\left(h \omega t_{j}\right)\right)\right]
\end{gathered}
$$

$$
\begin{gathered}
\frac{\partial I^{\prime \prime}\left(h, t_{j}\right)}{\partial V_{r_{n}}}= \\
\frac{1}{2 \omega Z_{n}}\left[-\frac{\cos \left((h+n) \omega t_{j}-\varphi_{n}\right)}{h+n}-\frac{\cos \left((h-n) \omega t_{j}-\varphi_{n}\right)}{h-n}-\right. \\
2 \omega \cos \left(n \omega t_{o n_{j}}-\varphi_{n}\right) \frac{e^{-\frac{R}{L}\left(t_{j}-t_{o n_{j}}\right)} L}{\left(Z_{h}\right)^{2}} \\
\left.\left(-R \operatorname{sen}\left(h \omega t_{j}\right)-h \omega L \cos \left(h \omega t_{j}\right)\right)\right]
\end{gathered}
$$




$$
\begin{gathered}
\frac{\partial I^{\prime \prime}\left(h, t_{j}\right)}{\partial V_{m_{n}}}= \\
\frac{1}{2 \omega Z_{n}}\left[\frac{\operatorname{sen}\left((n-h) \omega t_{j}-\varphi_{n}\right)}{n-h}-\frac{\operatorname{sen}\left((n+h) \omega t_{j}-\varphi_{n}\right)}{n+h}-\right. \\
2 \omega \operatorname{sen}\left(n \omega t_{o n_{j}}-\varphi_{n}\right) \frac{e^{-\frac{R}{L}\left(t_{j}-t_{o n_{j}}\right)} L}{\left(Z_{h}\right)^{2}} \\
\left.\left(-R \operatorname{sen}\left(h \omega t_{j}\right)-h \omega L \cos \left(h \omega t_{j}\right)\right)\right]
\end{gathered}
$$

Observe que a representação dos acoplamentos entre frequiências através das contribuições $\left[J_{k k}^{a b c}\right]_{h n} \mathrm{e}\left[J_{k k}^{a b c}\right]_{n h}$, fora da diagonal principal da matriz Jacobiana dada em (51), só é possível devido ao agrupamento dos subsistemas das frequiências simuladas em um único sistema matricial unificado e apresentado no presente trabalho.

O modelo proposto para o RCT trifásico permite que o mesmo seja simulado com todos os seus parâmetros desequilibrados (apesar de não praticado) e com ângulos de disparos assimétricos nos seis tiristores. Além disso, a inclusão da resistência em série com o indutor permite que as perdas do equipamento sejam devidamente representadas.

\subsection{Compensador Estático de Reativo}

O Compensador Estático de Reativo (CER) se comporta operacionalmente como uma reatância variável, conectada em derivação com os sistemas de potência, absorvendo ou gerando reativos em seus terminais. Além de possuir uma resposta rápida no suporte de reativos o CER possui a vantagem de não possuir partes mecânicas. O CER consiste basicamente de um RCT ligado em paralelo a um banco de capacitores, conforme ilustrado na Figura 4.

Existe também a opção dos bancos de capacitores serem chaveados por tiristores (CCT, Capacitor Chaveado por Tiristores). Contudo, neste caso, os tiristores são chaveados somente com tensão nula entre seus terminais. Esta característica não será explorada neste trabalho pois em regime permanente o chaveamento com tensão nula não gera harmônicos com o disparo dos semicondutores.

Baseado no diagrama esquemático do CER mostrado na Figura 4, a contribuição matricial no subsistema de freqüência $h$ para um compensador conectado em uma barra $k$ é dada pelas contribuições do RCT e do banco de capacitores, ou seja:

$$
\begin{gathered}
{\left[\Delta I_{k}^{a b c}\right]_{h}=-\left[I_{k}^{a b c}\right]_{h}^{c e r}=-\left[I_{k}^{a b c}\right]_{h}^{r c t}-\left[I_{k}^{a b c}\right]_{h}^{b c o}} \\
{\left[J_{k k}^{a b c}\right]_{h h}=\left[J_{k k}^{a b c}\right]_{h h}^{r c t}+\left[J_{k k}^{a b c}\right]_{h h}^{b c o}} \\
{\left[J_{k k}^{a b c}\right]_{h n}=\left[J_{k k}^{a b c}\right]_{h n}^{r c t}}
\end{gathered}
$$

onde:

$\left[I_{k}^{a b c}\right]_{h}^{c e r}$ é o vetor trifásico e complexo da corrente nodal consumida pelo CER na freqüência $h$;

$\left[I_{k}^{a b c}\right]_{h}^{r c t}$ é o vetor trifásico e complexo da corrente nodal consumida pelo RCT na freqüência $h$;

$\left[I_{k}^{a b c}\right]_{h}^{b c o}$ é o vetor trifásico complexo da corrente nodal consumida pelo banco de capacitores na freqüência $h$;

$\left[J_{k k}^{a b c}\right]_{h h}^{r c t}$ é a matriz Jacobiana trifásica complexa representando a contribuição do RCT no subsistema de freqüências $h$;

$\left[J_{k k}^{a b c}\right]_{h h}^{b c o}$ é a matriz Jacobiana trifásica complexa representando a contribuição do banco de capacitores no subsistema de frequiências $h$;

$\left[J_{k k}^{a b c}\right]_{h n}^{r c t}$ é a matriz Jacobiana trifásica complexa representando os acoplamentos entre as freqüências $h$ e $n$.

Note que as contribuições matriciais do CER são análogas àquelas dadas por (51), entretanto com os resíduos de corrente calculados por (68) e matriz Jacobiana calculado por (69) e (70).

Por se tratar de um dispositivo de chaveamento, o CER é uma fonte harmônica, cuja geração de componentes harmônicos é dependente do conteúdo harmônico da tensão do barramento

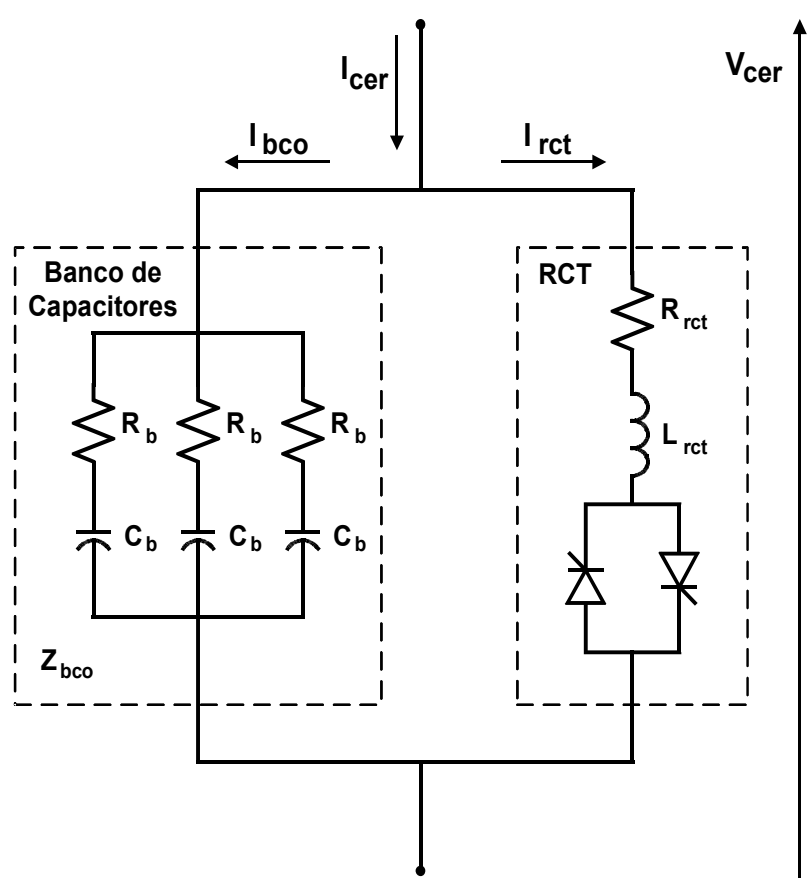

Figura 4: Representação esquemática do CER. 


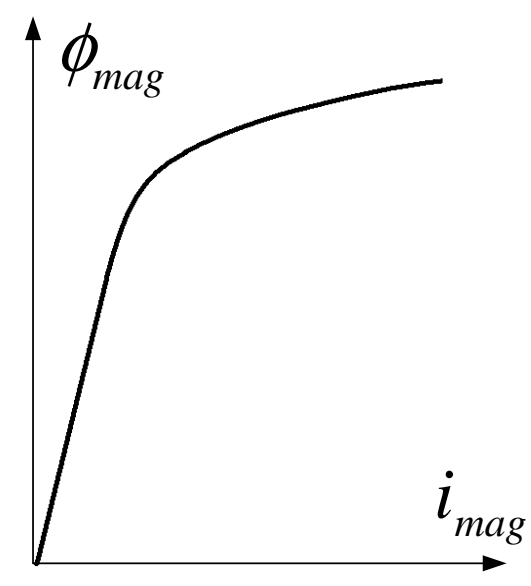

Figura 5: Curva de magnetização típica de um transformador.

ao qual está conectado. Note que o CER é composto por dois blocos distintos, um com característica linear (banco de capacitores) e outro não-linear (RCT). Como estes blocos são representados modularmente, o modelo do CER possibilita que as conexões do RCT e do banco de capacitores sejam distintas entre si.

\subsection{Transformador Saturado}

O transformador apresenta um comportamento não-linear devido às características do seu circuito magnético, conforme pode ser observado pela curva de magnetização típica ilustrada na Figura 5.

O fluxo magnético nos enrolamentos de um transformador em função da tensão aplicada é expresso por:

$$
\phi_{m a g}(t)=\int v(t) d t
$$

Portanto, as não-linearidades do transformador operando com núcleo saturado afetam as características de corrente e tensão nos seus terminais, conforme observado na Figura 6.

O modelo proposto para a representação do transformador de potência saturado é apresentado na Figura 7. As impedâncias $Z_{S e}, Z_{S h p}$ e $Z_{S h s}$ são constituídas pelas resistências dos enrolamentos primário e secundário, pelas indutâncias de dispersão, e pela resistência que representa as perdas no núcleo.

O modelo apresentado (Figura 7) é formado por duas partes, uma representando o comportamento não-linear da corrente de magnetização, e outra caracterizada pelos componentes passivos constituídos por $Z_{S e}, Z_{S h p}$ e $Z_{S h s}$.

Portanto, a corrente nos terminais do transformador para uma freqüência harmônica $h$ é dada pelo somatório das correntes de ambas as partes, ou seja:

$$
I_{h}^{\text {trafo }}=I_{h}^{\text {mag }}+I_{h}^{\text {linear }}
$$

Considerando que a tensão nos terminais do transformador é distorcida e expressa pela sua série de Fourier correspondente, conforme mostrado em (42), tem-se de (71):

$$
\phi_{m a g}(t)=\sum_{n=1}^{\infty}\left(\frac{V_{r_{n}}}{n \omega} \operatorname{sen}(n \omega t)-\frac{V_{m_{n}}}{n \omega} \cos (n \omega t)\right)
$$

A representação matemática das características não-lineares da corrente de magnetização é obtida utilizando o polinômio interpolador de Newton a partir dos pontos conhecidos da curva de magnetização do transformador (Figura 5) (Variz et alii, 2007). Estes pontos são obtidos através de ensaios à vazio ou fornecidos pelo fabricante.

Uma interpolação satisfatória para a curva de magnetização é obtida utilizando quatro ou mais pontos no quadrante positivo $\left(\varnothing_{\text {mag }}(t)>0\right)$. Na modelagem apresentada neste trabalho foram utilizados cinco pontos. Desta forma, a corrente de magnetização no quadrante positivo é calculada por:

$i_{m a g}=P_{5} \phi_{m a g}^{5}+P_{4} \phi_{m a g}^{4}+P_{3} \phi_{m a g}^{3}+P_{2} \phi_{m a g}^{2}+P_{1} \phi_{m a g}+P_{0}$

onde $P_{i}$ é o $i$-ésimo coeficiente do polinômio de Newton, cujo cálculo é apresentado no Apêndice A.

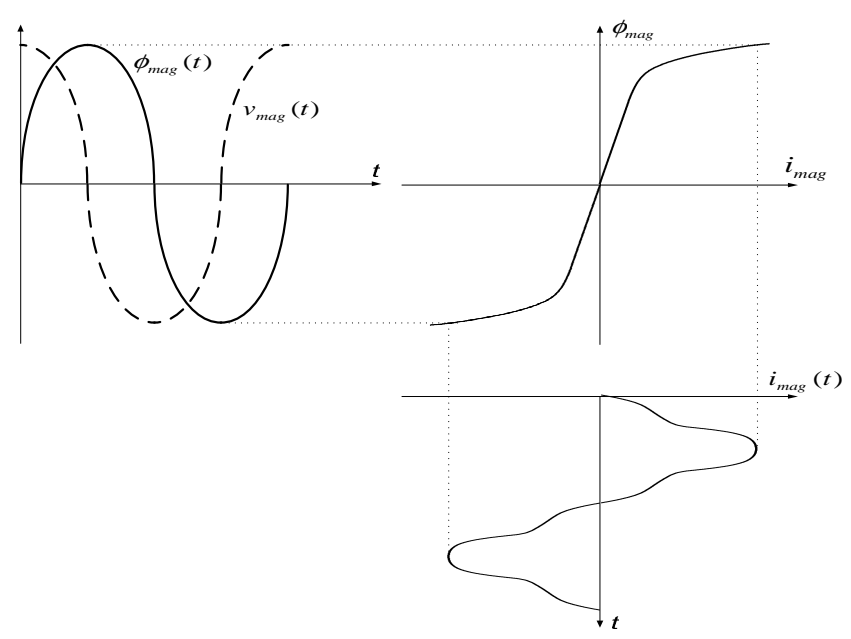

Figura 6: Característica de tensão e corrente do transformador operando com núcleo saturado. 


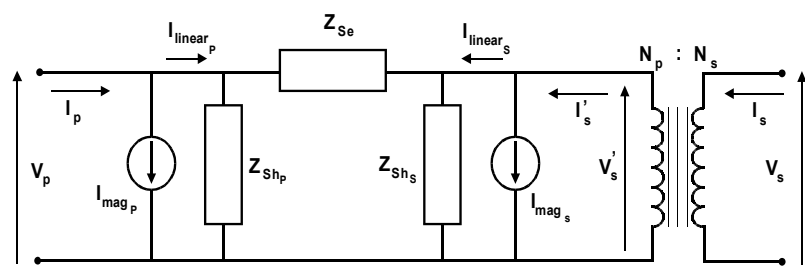

Figura 7: Modelo proposto para o transformador.

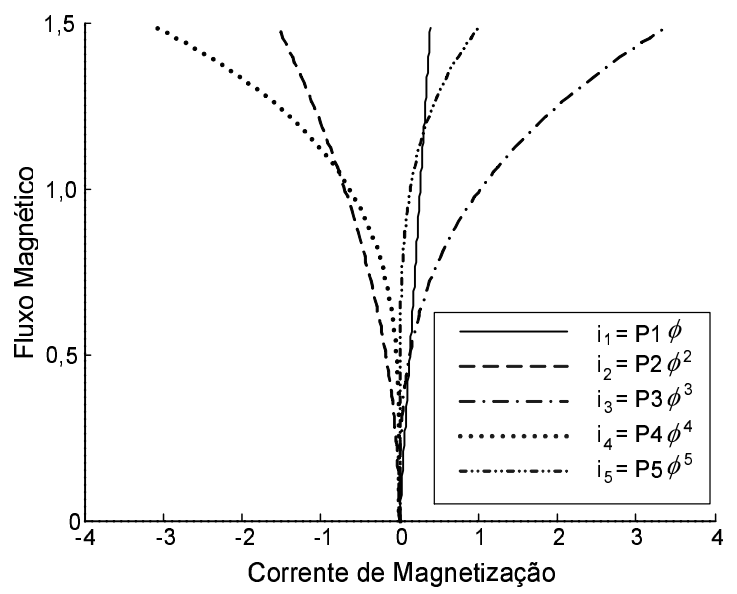

Figura 8: Interpolação de Newton de uma curva de magnetização típica.

A composição da corrente de magnetização dada em (74) é formada pelo somatório de um conjunto de curvas conforme mostrado na Figura 8.

Com a finalidade de reduzir a complexidade do modelo, os efeitos da histerese foram desprezados no presente trabalho. Conseqüentemente, a curva de magnetização será simétrica com zero na origem, $P_{0}=0$ em (74). Desta forma, as correntes de magnetização nos quadrantes positivo e negativo são dadas respectivamente por:

$$
\begin{aligned}
i_{\text {mag }}^{+}(t)= & P_{5} \phi_{\text {mag }}^{5}(t)+P_{4} \phi_{\text {mag }}^{4}(t)+ \\
& P_{3} \phi_{\text {mag }}^{3}(t)+P_{2} \phi_{\text {mag }}^{2}(t)+P_{1} \phi_{\text {mag }}(t) \\
i_{\text {mag }}^{-}(t)= & P_{5} \phi_{\text {mag }}^{5}(t)-P_{4} \phi_{\text {mag }}^{4}(t)+ \\
& P_{3} \phi_{\text {mag }}^{3}(t)-P_{2} \phi_{\text {mag }}^{2}(t)+P_{1} \phi_{\text {mag }}(t)
\end{aligned}
$$

onde:
Portanto:

$$
\begin{aligned}
i_{\text {mag }}(t)= & P_{5} \phi_{\text {mag }}^{5}(t) \pm P_{4} \phi_{\text {mag }}^{4}(t)+ \\
& P_{3} \phi_{\text {mag }}^{3}(t) \pm P_{2} \phi_{\text {mag }}^{2}(t)+P_{1} \phi_{\text {mag }}(t)
\end{aligned}
$$

Expandindo (77) em série de Fourier, tem-se as seguintes componentes real e imaginária da corrente de magnetização para uma dada freqüência harmônica $h$ :

$$
\begin{aligned}
& I_{t r f r_{h}}=\frac{\omega}{\pi}\left[P_{5} F_{r_{5}}(h)+P_{4} F_{r_{4}}(h)+\right.\left.P_{3} F_{r_{3}}(h)+P_{2} F_{r_{2}}(h)+P_{1} F_{r_{1}}(h)\right] \\
& I_{t r f m_{h}}=\frac{\omega}{\pi}\left[P_{5} F_{m_{5}}(h)+P_{4} F_{m_{4}}(h)+\right. \\
&\left.P_{3} F_{m_{3}}(h)+P_{2} F_{m_{2}}(h)+P_{1} F_{m_{1}}(h)\right]
\end{aligned}
$$

onde para valores ímpares de $j(j=1,3,5)$ tem-se:

$$
\begin{aligned}
& F_{r_{j}}(h)=\int_{0}^{T} \phi_{m}^{j}(t) \cos (h \omega t) d t=G_{j_{r}}(h, T)-G_{j_{r}}(h, 0) \\
& F_{m_{j}}(h)=\int_{0}^{T} \phi_{m}^{j}(t) \operatorname{sen}(h \omega t) d t=G_{j_{m}}(h, T)-G_{j_{m}}(h, 0)
\end{aligned}
$$

E para valores pares de $j(j=2,4)$ tem-se:

$$
\begin{aligned}
& F_{r_{j}}(h)=\int_{0}^{T / 2} \phi_{m}^{j}(t) \cos (h \omega t) d t-\int_{T / 2}^{T} \phi_{m}^{j}(t) \cos (h \omega t) d t \\
= & G_{j_{r}}(h, T / 2)-G_{j_{r}}(h, 0)-G_{j_{r}}(h, T)+G_{j_{r}}(h, T / 2)
\end{aligned}
$$

$$
\begin{aligned}
& F_{m_{j}}(h)=\int_{0}^{T / 2} \phi_{m}^{j}(t) \operatorname{sen}(h \omega t) d t-\int_{T / 2}^{T} \phi_{m}^{j}(t) \operatorname{sen}(h \omega t) d t \\
= & G_{j_{m}}(h, T / 2)-G_{j_{m}}(h, 0)-G_{j_{m}}(h, T)+G_{j_{m}}(h, T / 2)
\end{aligned}
$$

Substituindo-se (73) em (80) a (83), manipulando-se os somatórios e as funções trigonométricas e resolvendo-se as integrais têm-se as seguintes equações:

$$
\begin{aligned}
& i_{\text {mag }}(t)=i_{\text {mag }}^{+}(t), \quad \text { para } \phi_{\text {mag }}(t)>0 \\
& i_{\text {mag }}(t)=i_{\text {mag }}^{-}(t), \quad \text { para } \quad \phi_{m a g}(t)<0
\end{aligned}
$$




$$
\begin{gathered}
G_{j_{r}}(h, t)=\sum_{n_{1}}^{\infty} \sum_{n_{2}}^{\infty} \ldots \sum_{n_{j}}^{\infty} \frac{1}{\left(n_{1} n_{2} \ldots n_{j}\right) \omega^{j+1}} \sum_{K}^{2^{j-1}}\{ \\
V_{j_{K}}^{\prime}\left(n_{1} \ldots n_{j}\right)\left[\frac{\cos \left(\left(\beta_{j_{K}}\left(n_{1} n_{2} \ldots n_{j}\right)-h\right) \omega t\right)}{\beta_{j_{K}}\left(n_{1} n_{2} \ldots n_{j}\right)-h}+\right. \\
\left.\frac{\cos \left(\left(\beta_{j_{K}}\left(n_{1} n_{2} \ldots n_{j}\right)+h\right) \omega t\right)}{\beta_{j_{K}}\left(n_{1} n_{2} \ldots n_{j}\right)+h}\right]+ \\
V_{j_{K}}^{\prime \prime}\left(n_{1} \ldots n_{j}\right)\left[\frac{\operatorname{sen}\left(\left(\beta_{j_{K}}\left(n_{1} n_{2} \ldots n_{j}\right)-h\right) \omega t\right)}{\beta_{j_{K}}\left(n_{1} n_{2} \ldots n_{j}\right)-h}+\right. \\
\left.\left.\frac{\operatorname{sen}\left(\left(\beta_{j_{K}}\left(n_{1} n_{2} \ldots n_{j}\right)+h\right) \omega t\right)}{\beta_{j_{K}}\left(n_{1} n_{2} \ldots n_{j}\right)+h}\right]\right\}
\end{gathered}
$$

$$
\begin{gathered}
G_{j_{m}}(h, t)=\sum_{n_{1}}^{\infty} \sum_{n_{2}}^{\infty} \ldots \sum_{n_{j}}^{\infty} \frac{1}{\left(n_{1} n_{2} \ldots n_{j}\right) \omega^{j+1}} \sum_{K}^{2^{j-1}}\{ \\
V_{j_{K}}^{\prime \prime}\left(n_{1} \ldots n_{j}\right)\left[\frac{\cos \left(\left(\beta_{j_{K}}\left(n_{1} n_{2} \ldots n_{j}\right)-h\right) \omega t\right)}{\beta_{j_{K}}\left(n_{1} n_{2} \ldots n_{j}\right)-h}-\right. \\
\left.\frac{\cos \left(\left(\beta_{j_{K}}\left(n_{1} n_{2} \ldots n_{j}\right)+h\right) \omega t\right)}{\beta_{j_{K}}\left(n_{1} n_{2} \ldots n_{j}\right)+h}\right]- \\
V_{j_{K}}^{\prime}\left(n_{1} \ldots n_{j}\right)\left[\frac{\operatorname{sen}\left(\left(\beta_{j_{K}}\left(n_{1} n_{2} \ldots n_{j}\right)-h\right) \omega t\right)}{\beta_{j_{K}}\left(n_{1} n_{2} \ldots n_{j}\right)-h}-\right. \\
\left.\left.\frac{\operatorname{sen}\left(\left(\beta_{j_{K}}\left(n_{1} n_{2} \ldots n_{j}\right)+h\right) \omega t\right)}{\beta_{j_{K}}\left(n_{1} n_{2} \ldots n_{j}\right)+h}\right]\right\}
\end{gathered}
$$

Onde as funções auxiliares $V_{j_{k}}^{\prime}\left(n_{1}, \ldots, n_{j}\right), V_{j_{k}}^{\prime \prime}\left(n_{1}, \ldots, n_{j}\right)$ e $\beta_{j_{K}}\left(n_{1}, \ldots, n_{j}\right)$ são apresentadas em detalhes em Variz (2006).

É importante observar que no cálculo da corrente de magnetização para uma dada frequiência $h$ através de (78) e (79), o somatório de tensões dadas em (84) e (85) são independentes da frequiência harmônica $h$.

Para um transformador saturado conectado entre as barras $i$ e $k$ do sistema, as contribuições matriciais do modelo proposto deste equipamento no MICTH nas freqüências $h$ e $n$ são dadas por:

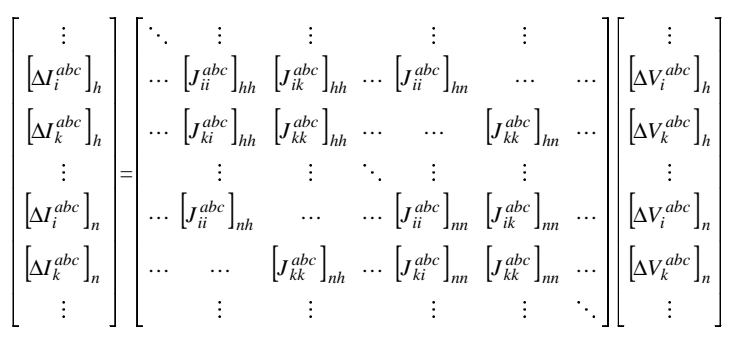

$$
\begin{gathered}
{\left[\Delta I_{k}^{a b c}\right]_{h}=-\left[I_{k}^{a b c}\right]_{h}^{\text {trf }}=-\left[I_{k}^{a b c}\right]_{h}^{\text {mag }}-\left[I_{k}^{a b c}\right]_{h}^{\text {linear }}} \\
{\left[J_{k k}^{a b c}\right]_{h h}=\left[J_{k k}^{a b c}\right]_{h h}^{\text {mag }}+\left[J_{k k}^{a b c}\right]_{h h}^{\text {linear }}} \\
{\left[J_{k i}^{a b c}\right]_{h h}=\left[J_{k i}^{a b c}\right]_{h h}^{\text {linear }}} \\
{\left[J_{k k}^{a b c}\right]_{h n}=\left[J_{k k}^{a b c}\right]_{h n}^{\text {mag }}}
\end{gathered}
$$

$\left[I_{k}^{a b c}\right]_{h}^{\operatorname{trf}}$ é o vetor trifásico e complexo com a corrente nodal do transformador na frequiência $h$;

$\left[I_{k}^{a b c}\right]_{h}^{\text {mag }}$ é o vetor trifásico e complexo com a corrente de magnetização na freqüência $h$ calculado pelas equações (78) e (79);

$\left[I_{k}^{a b c}\right]_{h}^{l i n e a r}$ é o vetor trifásico e complexo com a corrente da parte linear do transformador na freqüência $h$;

$\left[J_{k k}^{a b c}\right]_{h h}^{m a g}$ é a matriz Jacobiana trifásica e complexa representando a contribuição da parte não-linear do transformador no subsistema de frequiência $h$;

$\left[J_{k i}^{a b c}\right]_{h h}^{l i n e a r}$ é a matriz Jacobiana trifásica e complexa representando a contribuição da parte linear do transformador no subsistema de frequiência $h$;

$\left[J_{k k}^{a b c}\right]_{h n}^{m a g}$ é a matriz Jacobiana trifásica complexa representando os acoplamentos harmônicos entre as freqüências $h$ e $n$. Este acoplamento é devido à característica nãolinear da corrente de magnetização.

As contribuições da parte não-linear na matriz Jacobiana são calculadas pelas derivadas parciais de (78) e (79) em relação à tensão, portanto, formadas pelas derivadas parciais de (84) e (85) (Variz, 2006). Por outro lado, as contribuições da parte linear são obtidas pela matriz admitância nodal formada pelas resistências dos enrolamentos, indutâncias de dispersão e resistência representativa das perdas no núcleo.

Note que devido às características não-lineares da corrente de magnetização, o modelo do transformador apresenta acoplamentos harmônicos, conforme observado nos blocos fora da diagonal principal em (86).

É importante ressaltar que a contribuição final do transformador no sistema matricial do MICTH a cada iteração deverá ser ajustada em função do tipo de conexão dos terminais do equipamento, conforme apresentado por Chen e Dillon (1974) com o uso da matriz de incidência nodal de Kron.

Apesar do grande volume de dados calculados a cada iteração, existem diversas similaridades que levam a simplificações significativas nos cálculos dos somatórios. 


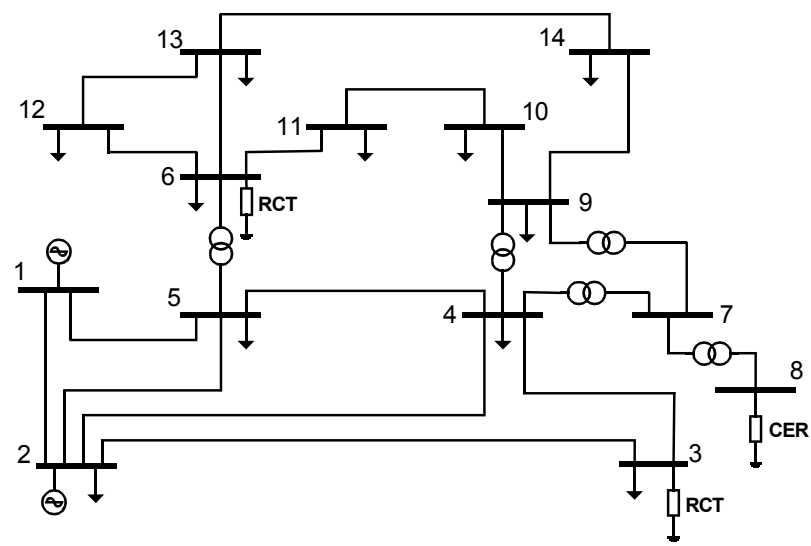

Figura 9: Sistema teste IEEE 14 barras modificado.

\section{RESULTADOS}

A metodologia proposta (MICTH) foi implementada computacionalmente em $\mathrm{C}++$ utilizando programação visual e orientada a objetos. A validação do MICTH foi realizada através de simulações comparativas com os resultados obtidos por simulações no domínio do tempo realizadas com o programa ATP/EMTP (Alternative Transients ProgramElectromagnetic Transients Program).

O sistema teste utilizado foi o IEEE 14 barras (IEEE, 1996) adaptado para análise harmônica com a inclusão de componentes trifásicos, cargas desequilibradas, RCTs, CER e transformadores saturados. O sistema teste é ilustrado na Figura 9 e apresentado em detalhes no Apêndice B. Com o intuito de explorar a total propagação dos componentes harmônicos pela rede, as fontes harmônicas do sistema composta pelos RCTs, CER e transformadores saturados foram simulados sem a inclusão de filtros. Apesar dos RCTs serem normalmente conectados em delta para a eliminação de harmônicas de terceira ordem, nesse estudo o RCT conectado na barra 3 e o CER conectado na barra 8 foram conectados em estrela.

O sistema teste foi simulado duas vezes. A primeira com todos os transformadores operando na região não-saturada, e segunda com os transformadores conectados entre as barras 4 e 7 , e entre as barras 5 e 6 operando com o núcleo saturado. Os resultados apresentados pelo MICTH foram obtidos simulando-se as 30 primeiras freqüências harmônicas características. A convergência do MICTH em ambas as simulações é alcançada em 6 iterações com resíduos de corrente nodal menores que $10^{-4}$ p.u.

A Tabela 1 apresenta as tensões harmônicas e a DHT (Distorção Harmônica Total) (IEEE, 1993) na barra 6 com os transformadores operando sem saturação. Além dos resultados comparativos entre o MICTH e o ATP, são incluídos resul- tados calculados pelo método de injeção de corrente monofásico baseada em solução direta e desacoplada (Arrillaga $e t$ alii, 1985), cujos dados de injeções harmônicas são obtidos a partir dos resultados de seqüência positiva do MICTH.

Tabela 1: Tensões harmônicas (em p.u.) na barra 6.

\begin{tabular}{|c|c|c|c|c|c|c|c|}
\hline & \multicolumn{2}{|c|}{ Fase A } & \multicolumn{2}{c|}{ Fase B } & \multicolumn{2}{c|}{ Fase C } & Equiv. \\
\hline$h$ & MICTH & ATP & MICTH & ATP & MICTH & ATP & Monofas. \\
\hline 1 & 1,0290 & 1,0247 & 1,0138 & 1,0160 & 1,0054 & 1,0010 & 1,0775 \\
\hline 3 & 0,0385 & 0,0391 & 0,0100 & 0,0102 & 0,0535 & 0,0576 & 0,0255 \\
\hline 5 & 0,0330 & 0,0306 & 0,0361 & 0,0340 & 0,0147 & 0,0139 & 0,0049 \\
\hline 7 & 0,0334 & 0,0359 & 0,0281 & 0,0292 & 0,0096 & 0,0098 & 0,0208 \\
\hline 9 & 0,0090 & 0,0075 & 0,0015 & 0,0014 & 0,0042 & 0,0040 & 0,0031 \\
\hline 11 & 0,0060 & 0,0066 & 0,0161 & 0,0166 & 0,0113 & 0,0123 & 0,0047 \\
\hline DHT & $6,24 \%$ & $6,28 \%$ & $5,10 \%$ & $5,01 \%$ & $5,86 \%$ & $6,26 \%$ & $3,27 \%$ \\
\hline
\end{tabular}

Os componentes harmônicos e a DHT da corrente injetada pelo RCT nesta barra calculados pelos três métodos (MICT, ATP e Equivalente Monofásico) são mostrados na Tabela 2.

Tabela 2: Corrente injetada (em p.u.) pelo RCT na barra 6.

\begin{tabular}{|c|c|c|c|c|c|c|c|}
\hline & \multicolumn{2}{|c|}{ Fase A } & \multicolumn{2}{c|}{ Fase B } & \multicolumn{2}{c|}{ Fase C } & Equiv. \\
\hline$h$ & MICTH & ATP & MICTH & ATP & MICTH & ATP & Monofas. \\
\hline 1 & 0,5510 & 0,5765 & 0,5462 & 0,5632 & 0,4919 & 0,5070 & 0,5291 \\
\hline 3 & 0,0640 & 0,0658 & 0,0208 & 0,0221 & 0,0472 & 0,0505 & 0,0389 \\
\hline 5 & 0,0556 & 0,0514 & 0,0653 & 0,0623 & 0,0710 & 0,0673 & 0,0089 \\
\hline 7 & 0,0486 & 0,0522 & 0,0420 & 0,0440 & 0,0303 & 0,0310 & 0,0396 \\
\hline 9 & 0,0111 & 0,0091 & 0,0021 & 0,0021 & 0,0093 & 0,0088 & 0,0055 \\
\hline 11 & 0,0061 & 0,0068 & 0,0175 & 0,0184 & 0,0167 & 0,0183 & 0,0067 \\
\hline DHT & $18,12 \%$ & $17,39 \%$ & $15,24 \%$ & $14,62 \%$ & $18,95 \%$ & $18,26 \%$ & $10,92 \%$ \\
\hline
\end{tabular}

Note que apesar do RCT estar conectado em delta, harmônicos de terceira ordem não são nulos devido aos desequilíbrios de fase da rede.

Como esperado não existem harmônicas de ordem ímpar, devido aos disparos dos RCTs e do CER serem todos simétricos.

Na Tabela 3 são apresentadas as distorções harmônicas totais de tensão nas barras do sistema com os transformadores operando sem saturação. Todas as DHTs foram obtidas considerando-se as 30 primeiras harmônicas. Pode-se conferir nesta tabela, a propagação harmônica pela rede e como esta afeta a qualidade da energia do sistema.

Observe que os resultados calculados pelo MICTH são bem próximos dos calculados pelo ATP, com diferenças justificá- 
Tabela 3: Distorção harmônica total (DHT) de tensão no sistema.

\begin{tabular}{|c|c|c|c|c|c|c|c|}
\hline & \multicolumn{2}{|c|}{ Fase A } & \multicolumn{2}{c|}{ Fase B } & \multicolumn{2}{c|}{ Fase C } & Equiv. \\
\hline Bar. & MICTH & ATP & MICTH & ATP & MICTH & ATP & Monofas. \\
\hline 1 & $0,00 \%$ & $0,00 \%$ & $0,00 \%$ & $0,00 \%$ & $0,00 \%$ & $0,00 \%$ & $0,00 \%$ \\
\hline 2 & $0,00 \%$ & $0,00 \%$ & $0,00 \%$ & $0,00 \%$ & $0,00 \%$ & $0,00 \%$ & $0,00 \%$ \\
\hline 3 & $1,57 \%$ & $1,50 \%$ & $0,85 \%$ & $0,83 \%$ & $1,60 \%$ & $1,63 \%$ & $0,69 \%$ \\
\hline 4 & $1,66 \%$ & $1,65 \%$ & $1,10 \%$ & $1,04 \%$ & $1,53 \%$ & $1,59 \%$ & $0,94 \%$ \\
\hline 5 & $1,93 \%$ & $1,94 \%$ & $1,43 \%$ & $1,34 \%$ & $1,63 \%$ & $1,70 \%$ & $1,10 \%$ \\
\hline 6 & $6,24 \%$ & $6,28 \%$ & $5,10 \%$ & $5,01 \%$ & $5,86 \%$ & $6,26 \%$ & $3,27 \%$ \\
\hline 7 & $2,17 \%$ & $2,08 \%$ & $1,63 \%$ & $1,52 \%$ & $2,52 \%$ & $2,61 \%$ & $1,13 \%$ \\
\hline 8 & $2,87 \%$ & $2,68 \%$ & $4,06 \%$ & $3,52 \%$ & $3,48 \%$ & $3,06 \%$ & $1,14 \%$ \\
\hline 9 & $2,75 \%$ & $2,69 \%$ & $2,01 \%$ & $1,90 \%$ & $3,22 \%$ & $3,39 \%$ & $1,48 \%$ \\
\hline 0 & $3,22 \%$ & $3,18 \%$ & $2,46 \%$ & $2,33 \%$ & $3,59 \%$ & $3,80 \%$ & $1,74 \%$ \\
\hline 11 & $4,59 \%$ & $4,59 \%$ & $3,69 \%$ & $3,56 \%$ & $4,63 \%$ & $4,94 \%$ & $2,45 \%$ \\
\hline 12 & $5,64 \%$ & $5,67 \%$ & $4,03 \%$ & $3,93 \%$ & $6,63 \%$ & $7,05 \%$ & $3,04 \%$ \\
\hline 13 & $5,66 \%$ & $5,68 \%$ & $3,68 \%$ & $3,58 \%$ & $7,74 \%$ & $8,14 \%$ & $3,25 \%$ \\
\hline 14 & $3,81 \%$ & $3,78 \%$ & $2,64 \%$ & $2,53 \%$ & $5,09 \%$ & $5,36 \%$ & $2,20 \%$ \\
\hline
\end{tabular}

veis pelo uso de métodos matemáticos totalmente distintos. Ao contrário do método de injeção monofásica com solução direta, onde os resultados diferem substancialmente devido aos desequilíbrios da rede.

Com os transformadores conectados entre as barras 4 e 7 , e entre as barras 5 e 6 operando com o núcleo saturado, têm-se os componentes harmônicos de tensão e o DHT nos terminais do transformador ligado entre as barras 4 e 7 apresentados nas Tabelas 4 e 5, respectivamente.

Tabela 4: Tensões harmônicas (em p.u.) na barra 4.

\begin{tabular}{|c|c|c|c|c|c|c|}
\hline & \multicolumn{2}{|c|}{ Fase A } & \multicolumn{2}{c|}{ Fase B } & \multicolumn{2}{c|}{ Fase C } \\
\hline$h$ & MICTH & ATP & MICTH & ATP & MICTH & ATP \\
\hline 1 & 0,9736 & 0,9764 & 0,9594 & 0,9624 & 0,9477 & 0,9488 \\
\hline 3 & 0,0433 & 0,0391 & 0,0260 & 0,0237 & 0,0334 & 0,0320 \\
\hline 5 & 0,0212 & 0,0149 & 0,0147 & 0,0112 & 0,0180 & 0,0145 \\
\hline 7 & 0,0069 & 0,0047 & 0,0072 & 0,0040 & 0,0050 & 0,0019 \\
\hline 9 & 0,0013 & 0,0048 & 0,0008 & 0,0041 & 0,0006 & 0,0047 \\
\hline 11 & 0,0020 & 0,0031 & 0,0017 & 0,0020 & 0,0029 & 0,0031 \\
\hline DHT & $5,08 \%$ & $4,48 \%$ & $3,27 \%$ & $2,97 \%$ & $4,08 \%$ & $3,85 \%$ \\
\hline
\end{tabular}

Note que os resultados obtidos pelo MICTH e pelo ATP continuam próximos, porém ligeiramente superiores aos calculados sem a presença de saturação no núcleo dos transforma-
Tabela 5: Tensões harmônicas (em p.u.) na barra 7.

\begin{tabular}{|c|c|c|c|c|c|c|}
\hline & \multicolumn{2}{|c|}{ Fase A (p.u.) } & \multicolumn{2}{c|}{ Fase B (p.u.) } & \multicolumn{2}{c|}{ Fase C (p.u.) } \\
\hline$h$ & MICTH & $A T P$ & MICTH & ATP & MICTH & ATP \\
\hline 1 & 1,0055 & 1,0106 & 0,9740 & 0,9808 & 0,9517 & 0,9507 \\
\hline 3 & 0,0619 & 0,0541 & 0,0255 & 0,0238 & 0,0409 & 0,0438 \\
\hline 5 & 0,0269 & 0,0209 & 0,0193 & 0,0161 & 0,0232 & 0,0214 \\
\hline 7 & 0,0084 & 0,0044 & 0,0088 & 0,0025 & 0,0043 & 0,0005 \\
\hline 9 & 0,0021 & 0,0060 & 0,0011 & 0,0039 & 0,0011 & 0,0054 \\
\hline 11 & 0,0009 & 0,0024 & 0,0010 & 0,0021 & 0,0009 & 0,0023 \\
\hline DHT & $6,79 \%$ & $5,82 \%$ & $3,42 \%$ & $3,04 \%$ & $4,97 \%$ & $5,19 \%$ \\
\hline
\end{tabular}
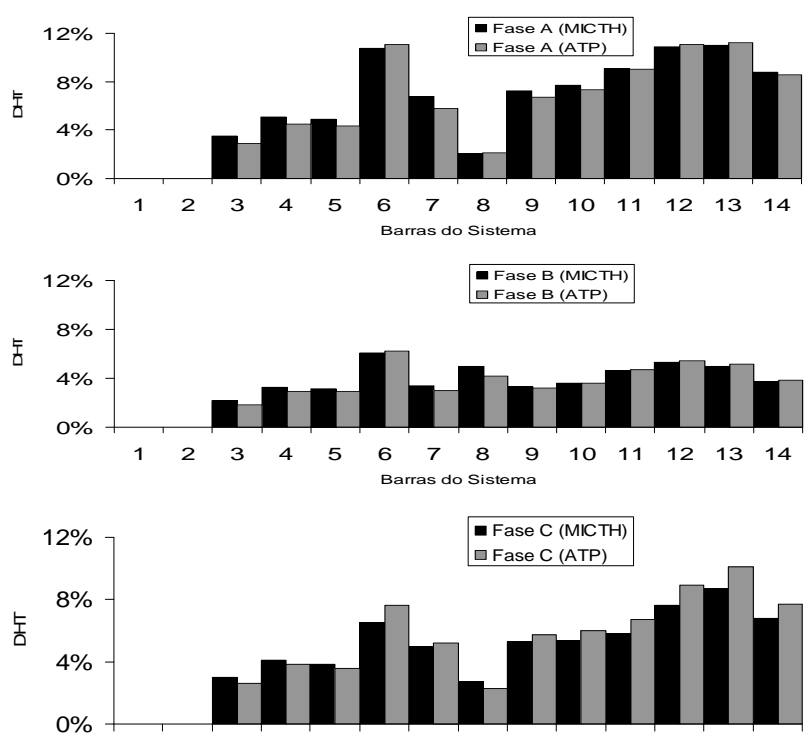

Figura 10: DHT de Tensão nas três fases.

dores. Esta característica é explicada pelo uso de modelos representativos e métodos de interpolação totalmente distintos na representação da curva de saturação do transformador em ambas as metodologias.

A propagação harmônica das distorções geradas pelos transformadores saturados, RCTs e CER é observada pelas DHTs de tensão em todas as barras do sistema ilustradas na Figura 10 .

Na Figura 11 são comparadas as DHTs do sistema com os transformadores operando na região saturada e não-saturada. Conforme esperado, com os transformadores operando saturados, a DHT é maior.

Com o intuito de analisar o desempenho computacional da metodologia proposta, a Tabela 6 apresenta os tempos computacionais gastos pelo MICTH para alcançar a convergên- 

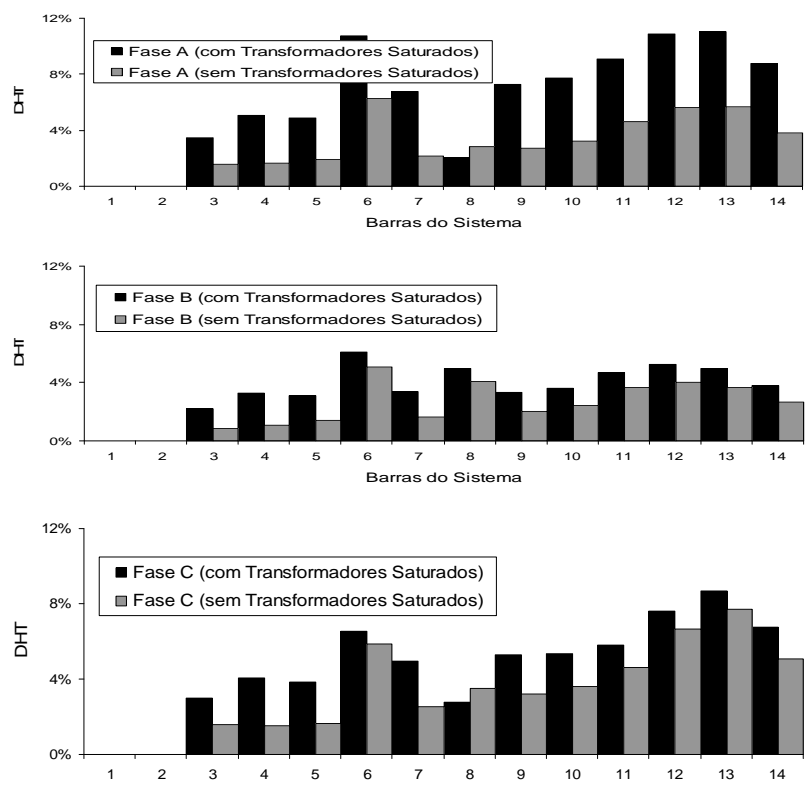

Figura 11: DHT de Tensão com transformadores saturados e não saturados.

cia na simulação de $H$ freqüências harmônicas. Em todas as simulações a convergência é obtida em 6 iterações com resíduos de corrente menores que $10^{-4}$ p.u. O sistema computacional utilizado para a análise é formado por um Pentium IV 2,4GHz, com $1 \mathrm{~GB}$ de RAM operando com MS Windows $\mathrm{XP} \operatorname{sp} 2$.

Tabela 6: Tempo computacional gasto pelo MICTH para alcançar a convergência na simulação de $H$ freqüências.

\begin{tabular}{|c|c|c|}
\hline$H$ & $\begin{array}{c}\text { Sistema teste com } \\
\text { trafos não saturados }\end{array}$ & $\begin{array}{c}\text { Sistema teste com } \\
\text { trafos saturado }\end{array}$ \\
\hline 15 & $0,266 \mathrm{~s}$ & $2,375 \mathrm{~s}$ \\
\hline 30 & $1,000 \mathrm{~s}$ & $4,888 \mathrm{~s}$ \\
\hline 45 & $2,703 \mathrm{~s}$ & $9,813 \mathrm{~s}$ \\
\hline 60 & $5,266 \mathrm{~s}$ & $12,75 \mathrm{~s}$ \\
\hline
\end{tabular}

Conforme esperado, o tempo computacional gasto cresce à medida que o número de freqüências simuladas aumenta. Isto ocorre porque o aumento do número de frequiências simuladas acarreta no aumento da dimensão do sistema matricial unificado, conseqüentemente, há um aumento no esforço computacional para a alocação de memória e solução do sistema de equações. Entretanto, é importante destacar que a precisão dos modelos e da metodologia é dependente do número de freqüências simuladas. Os tempos gastos na solução do sistema teste com transformadores operando saturados são superiores devido à complexidade da metodo- logia utilizada para a representação das características nãolineares do transformador. O tempo computacional gasto pelo ATP/EMTP para atingir o regime permanente de operação nas simulações do caso teste foi de aproximadamente 120 s. É importante destacar que este tempo depende de parâmetros de difícil definição como condições iniciais da rede, passo de integração e tempo final de simulação. A comparação desse tempo com aqueles apresentados na Tabela 6 fornece uma estimativa da ordem de grandeza associada ao esforço computacional entre o MICTH e o ATP/EMTP para solução do caso teste.

\section{CONCLUSÃo}

Este trabalho apresentou uma metodologia matemática para o cálculo de harmônicos em sistemas de potência trifásicos desequilibrados (MICTH). Os resultados obtidos são muito próximos aqueles obtidos por métodos no domínio do tempo (ATP), porém com menor esforço computacional devido ao uso de métodos de solução iterativa Newton-Raphson e formulação baseada em equações de injeção de corrente (atualização de poucos elementos do Jacobiano).

Os resultados obtidos com o MICTH mostraram que as metodologias monofásicas baseadas em métodos diretos de solução (Arrillaga et alii, 1985), embora bastante utilizadas, não apresentam resultados satisfatórios em redes com presença de dispositivos não-lineares e com cargas desequilibradas.

Embora não abordado durante as simulações, o MICTH permite a análise e a quantificação de componentes harmônicos não-característicos, como as inter-harmônicas e as subharmônicas, bastando incluir no sistema matricial unificado os subsistemas referentes às freqüências não-múltiplas inteiras da fundamental que se deseja investigar.

A representação do comportamento da rede e dos seus dispositivos só foi alcançada pelo MICTH, por este apresentar uma formulação trifásica em componentes de fase com o uso de um sistema matricial unificado.

Apesar do MICTH apresentar um sistema unificado com tamanho elevado na simulação de sistemas de dimensões reais e de grande porte, o esforço computacional não é consideravelmente alto, devido ao uso de eficientes ferramentas computacionais como técnicas de esparsidade, programação orientada a objetos e linguagem de programação para compiladores de alto desempenho. Portanto, a metodologia proposta permite uma análise sistêmica da propagação harmônica. Em comparação com o ATP/EMTP, o MICTH permite que um número maior de barras seja incluído nas simulações.

Por apresentar uma metodologia precisa e eficiente, o MICTH é compatível com a análise harmônica de sistemas 
elétricos de potência reais, principalmente em situações onde o uso de outras ferramentas é insatisfatório ou inviável, seja devido a incapacidade de simulação de componentes trifásicos desequilibrados, inabilidade na representação dos acoplamentos entre freqüências ou por restrições computacionais.

\section{AGRADECIMENTOS}

O autor Abilio M. Variz agradece ao professor Márcio P. Vinagre (UFJF), à COPPE-UFRJ, à UFJF e ao CNPq e CAPES pelo apoio financeiro neste trabalho. Beneficiário de auxílio financeiro da CAPES - Brasil.

\section{REFERÊNCIAS}

Acha, E., Arrillaga, J., Medina, A. e Semlyen, A. (1989). General frame of reference for analysis of harmonic distortion in systems with multiple transformer nonlinearities, Proc. Inst. Elect. Eng. C (Sept), vol.136, pp.271278.

Acha, E. e Madrigal, M. (2001). Power System Harmonics: Computer Modeling and Analysis. J. Wiley \& Sons, New York.

Arrillaga, J., Bradley, D.A. e Bodger, P.S. (1985). Power System Harmonics. J. Wiley \& Sons, New York.

Arrillaga, J., Smith, B.C., Watson, N.R. e Wood, A.R. (1997). Power System Harmonic Analysis. J. Wiley \& Sons, New York.

Arrillaga, J., Watson, N.R. e Bathurst, G.N. (2004). A Multifrequency Power Flow of General Applicability. IEEE Transactions on Power Delivery, vol.19, no.1, pp.342349.

Chen, M.S. e Dillon, W.E. (1974). Power System Modeling. Proceedings of the IEEE (Jul.), vol.62, no.7, pp.901915.

Chen, X. e Venkata, S. (1997). A three-phase three-winding core-type transformer model for low-frequency transient studies. IEEE Trans. Power Delivery (Apr.), vol.12, pp.775-782.

Da Costa, V.M., Martins, N. e Pereira, J.L.R. (1999). Developments in the Newton Raphson power flow formulation based on current injections. IEEE Transactions on Power Systems, vol.14, no.4, pp.1320-1326.

De Leon, F. e Semlyen, A. (1994). Complete transformer model for electromagnetic transients. IEEE Trans. Power Delivery (Jan.), vol. 9, pp. 231-239.
Domijan, A., Heydt, G.T., Meliopoulos, A.P.S., Venkata, S. e West, S. (1993). Directions in Research in Electric Power Quality. IEEE Transactions on Power Delivery, vol.8, pp.429-436.

Dommel, H.W. (1986). Electromagnetic Transients Program Reference Manual (EMTP Theorycal Book). Prepared for Bonneville Power Administration, Dept. of Electrical Engineering, University of British Columbia.

Dommel, H.W., Yan, A. e Shi, W. (1986). Harmonics from Transformer Saturation. IEEE Trans. On Power Delivery (Apr.), vol.PWRD-1, pp.209-215.

Dufeey, C.K. e Stratford, R.P. (1989). Update of harmonic standard IEEE-519: Recommended practices and requirements for harmonic control in electric power systems. IEEE Transactions on Industry and Applications, vol.25, no.6, pp.1025-1033.

Dugui, W. e Zheng, X. (1998). Harmonic model of power transformer, In Proc. POWERCON '98, International Conference on Power System Technology (Aug.), Beijing, China, pp.1045-1049.

Garcia, P.A.N., Pereira, J.L.R., Carneiro Jr., S., Da Costa, V.M. e Martins, N. (2000). Three-phase power flow calculations using the Current Injection Method. IEEE Transactions on Power Systems, vol.15, no.2, pp.508514.

Garcia, P.A.N., Pereira, J.L.R. e Carneiro Jr., S. (2001a). Fluxo de Potência Trifásico por Injeção de Correntes: Parte 1 - Formulação Básica, Controle e Automação. Revista Brasileira de Controle \& Automação - SBA, Brasil, vol.12, no.3, pp.178-187.

Garcia, P.A.N., Pereira, J.L.R. e Carneiro Jr., S. (2001b). Fluxo de Potência Trifásico por Injeção de Corrente: Parte 2 - Controles e Dispositivos FACTS. Revista Brasileira de Controle \& Automação - SBA, Brasil, vol.12, no.3, pp.188-196.

Garcia, P.A.N., Pereira, J.L.R., Carneiro Jr., S., Vinagre, M.P. e Gomes, F.V. (2004). Improvements in the Representation of PV Buses on Three-Phase Distribution Power Flow. IEEE Transactions on Power Delivery, vol.19, no.2, pp.894-896.

IEEE. (1983). Working Group on Power System Harmonics, "Power System Harmonics: An Overview". IEEE Transactions on Power Apparatus e Systems, vol.pas-102, pp.2455-2460.

IEEE std. 519-1992. (1993). IEEE Recommend Practices and Requirements for Harmonic Control in Electrical Power Systems. IEEE, New York. 
IEEE Working Group. (1996). Task Force. "Modeling and simulation of the propagation of harmonics in electric power network, Part I and II". IEEE Transactions on Power Delivery, vol.11, no.1, pp.452-474.

Kulicke, B. (1979). Digital program NETOMAC zur Simulation Elecktromechanischer und Magnetischer Ausleighsvorgänge in Drehstromnetzen. Electrizitatwirstschaft, 78, pp.18-23.

Lathi, B. P. (2005). Linear Systems and Signals. Oxford University Press, Second Edition.

Lima, L.T.G., Semlyen, A. e Iravani, M.R. (2003). Harmonic domain periodic steady state modeling of power electronics apparatus: SVC and TCSC. IEEE Transactions on Power Delivery, vol.18, no.3, pp.960-967.

Lin, W.M., Zhan, T.S. e Tsay, M.T. (2004). MultipleFrequency Three-Phase Load Flow for Harmonic Analysis. IEEE Transactions on Power Systems, vol.19, no.2, pp.897-904.

Marinos, Z.A., Pereira, J.L.R. e Carneiro Jr., S. (1994). Fast harmonic power flow calculation using parallel processing. IEE Proc.-Gem. Transm. Distrib., vol.141, no.1, pp.27-32.

Medina, A. e Arrillaga, J. (1992). Simulation of multilimb power transformers in the harmonic domain. Proc. Inst. Elect. Eng. C (May), vol.139, pp.269-276.

Neves, W.L.A. e Dommel, H.W. (1993). On Modelling Iron Core Nonlinearities. IEEE Transactions on Power Systems (May), vol.8, no.2, pp.417-425.

Semlyen, A., Acha, E. e Arrillaga, J. (1987). Harmonic Norton equivalent for the magnetising branch of a transformer. Proc. Inst. Elect. Eng. C (Mar.), vol.134, pp.162169.

Semlyen, A., Acha, E. e Arrillaga, J. (1988). Newton type algorithms for the harmonic phasor analysis of non-linear power circuits in periodical steady state with special reference to magnetic non-linearities. IEEE Transactions on Power Delivery, vol.3, no.3, pp.1090-1098.

Semlyen, A. e Shlash, M. (2000). Principles of modular harmonic power flow methodology. IEE Proceedings Generation Transmission and Distribution, vol.147, no.1.

Smith, B.C., Arrilaga, J., Wood, A.R. e Watson, N.R. (1998). A review of Iterative Harmonic Analysis for AC-DC Power Systems. IEEE Transactions on Power Delivery, vol.13. no.1, pp.180-185.

Tinney, W.F e Hart, E.E. (1967). Power Flow Solution by Newton's Method. IEEE Transactions on Power Apparatus and Systems, vol.86, pp.1449-1456.
Variz, A.M., Da Costa, V.M. (2002). Novos Desenvolvimentos numa Formulação de Injeção de Corrente para Solução de Fluxo de Potência. Revista Brasileira de Controle \& Automação (SBA), vol.13, no.3, pp.298-306.

Variz, A.M., Da Costa, V.M., Pereira, J.L.R. e Martins, N. (2003). Improved representation of control adjustments into the Newton-Raphson power flow. International Journal of Electrical Power e Energy Systems, vol.25, no.7, pp.501-513.

Variz, A.M., Pereira, J.L.R., Carneiro Jr., S. e Barbosa, P.G. (2006a). Three-Phase Harmonic Power Flow Using the Current Injection Method. IEEE International Conference on Harmonics and Power Quality, 12th ICHPQ (Oct.), Cascais, Portugal.

Variz, A.M., Pereira, J.L.R., Carneiro Jr., S. e Barbosa, P.G. (2006b). Fluxo de Potência Harmônico Trifásico. XVI Congresso Brasileiro de Automática, CBA 2006, Salvador, Bahia, Brasil.

Variz, A.M. (2006). Cálculo do Fluxo de Harmônicas em Sistemas Trifásicos Utilizando o Método de Injeção de Correntes. Tese de D.Sc., COPPE-UFRJ, Rio de Janeiro, Brasil. Disponível em "www.pee.ufrj.br/teses/".

Variz, A.M., Pereira, J.L.R., Carneiro Jr., S. e Barbosa, P.G. (2007). Modelo de Transformador Saturado para Cálculo do Fluxo de Potência pelo Método de Injeção de Corrente. VII Congresso Brasileiro de Qualidade de Energia Elétrica, CBQEE 2007, Santos, SP, Brasil.

Wagner, V.E. (1993). Effects of harmonics on equipment. IEEE Transactions on Power Delivery, vol.8, no.2, pp.672-680.

Xia, D. e Heydt, G.T. (1982). Harmonic power flow studies, part I formulation and solution, part II implementation and practical application. IEEE Transactions on Power Apparatus and Systems, vol.PAS-101, pp.1257-1270.

Xu, W., Marti, J.R. e Dommel, H.W. (1991). A multiphase harmonic load flow solution technique. IEEE Transactions on Power Systems, vol.6, no.1, pp.174-182.

\section{APÊNDICE}

\section{A - Interpolação de Newton}

Dada uma sequiência de $n+1$ pontos distintos $\left(y_{i}, x_{i}\right)$ de uma curva $f$, onde $y_{i}=f(x i)$, tem-se o polinômio interpolador de Newton representado por:

$$
f_{n}(x)=P^{5} x+P^{4} x+P^{3} x+P^{2} x+P^{1} x+x
$$


como resultado da seguinte expressão:

$$
\begin{array}{r}
f_{n}(x)=y_{0}+D^{1} y_{0}\left(x-x_{0}\right)+\ldots+ \\
D^{n} y_{0}\left(x-x_{0}\right) \ldots\left(x-x_{n-1}\right)
\end{array}
$$

onde:

$$
D^{n} y_{i}=\frac{D^{n-1} y_{i+1}-D^{n-1} y_{i}}{x_{i+n}-x_{i}}
$$

\section{B - Sistema Teste}

A Figura 9 apresenta o sistema teste IEEE 14 barras modificado para simulação no presente trabalho. As cargas das barras 2, 3, 5, 9, 10, 11 e 13 são conectadas em estrela, enquanto que as das barras 4, 6, 12 e 14 são conectadas em delta. Todas as cargas são equilibradas com os dados apresentados em IEEE (1996), exceto as cargas conectadas às barras 3, 4, 9 e 13 cujo desequilíbrio é apresentado na Tabela 7.

Tabela 7: Dados das cargas desbalanceadas.

\begin{tabular}{|c|c|c|c|c|c|c|}
\hline & \multicolumn{2}{|c|}{ Fase A (p.u.) } & \multicolumn{2}{c|}{ Fase B (p.u.) } & \multicolumn{2}{c|}{ Fase C (p.u.) } \\
\hline Barr & $R$ & $X$ & $R$ & $X$ & $R$ & $X$ \\
\hline 3 & 1,331 & $-0,361$ & 0,531 & $-0,269$ & 1,314 & 0,000 \\
\hline 4 & 5,962 & 0,477 & 3,713 & 0,371 & 14,42 & 2,885 \\
\hline 9 & 9,615 & $-1,923$ & 4,988 & 1,880 & 9,901 & $-0,495$ \\
\hline 13 & 0,735 & $-2,261$ & 0,706 & $-0,824$ & 0,125 & $-2,494$ \\
\hline
\end{tabular}

O RCT da barra 3 é conectado em estrela, enquanto que o da barra 6 é conectado em delta. Os parâmetros deste equipamento são listados na Tabela 8.

Tabela 8: Dados dos RCTs.

\begin{tabular}{|c|c|c|c|}
\hline Barra & L (p.u.) & R (p.u.) & $\begin{array}{c}\text { Ângulo de } \\
\text { Disparo }\end{array}$ \\
\hline 3 & 0,00265 & 0,0010 & $160,0^{\circ}$ \\
\hline 6 & 0,00265 & 0,0010 & $135,0^{\circ}$ \\
\hline
\end{tabular}

Os dados do CER conectado em estrela na barra 8 são apresentados na Tabela 9.

Os dados dos transformadores são listados na Tabela 10.

Nas Tabelas 11 e 12 são listados os pontos utilizados pelo MICTH e pelo ATP/EMTP interpolar a curva de magnetização dos transformadores saturados conectados entre as barras 4 e 7 , e entre as barra 5 e 6 .
Tabela 9: Dados do CER.

\begin{tabular}{|c|c|c|c|c|}
\hline \multicolumn{2}{|c|}{$\begin{array}{c}\text { Banco de } \\
\text { Capacitores }\end{array}$} & \multicolumn{3}{|c|}{ Reator Controlado a Tiristor } \\
\hline$R($ p.u. $)$ & $C($ p.u. $)$ & $L($ p.u. $)$ & $R(p . u)$. & $\begin{array}{c}\text { Ângulo de } \\
\text { disparo }\left(^{\circ}\right)\end{array}$ \\
\hline 1,00 & 0,00265 & 0,00530 & 0,0010 & 150,0 \\
\hline
\end{tabular}

Tabela 10: Dados dos transformadores (em p.u.).

\begin{tabular}{|c|c|c|c|c|c|c|}
\hline De & Para & Conexão & Tap & $\begin{array}{c}\text { Resistência } \\
\text { enrola- } \\
\text { mento }\end{array}$ & $\begin{array}{c}\text { Indutância } \\
\text { dispersão }\end{array}$ & $\begin{array}{c}\text { Resistência } \\
\text { perdas no } \\
\text { núcleo }\end{array}$ \\
\hline 4 & 7 & Y-Y & 0,978 & 0,010 & 0,20912 & 100,0 \\
\hline 4 & 9 & Y-Y & 0,969 & 0,010 & 0,55618 & 100,0 \\
\hline 5 & 6 & Y-Y & 0,932 & 0,010 & 0,25201 & 100,0 \\
\hline 7 & 8 & Y- $\Delta$ & 0,600 & 0,010 & 0,17615 & 100,0 \\
\hline 7 & 9 & Y-Y & 1,000 & 0,010 & 0,11001 & 100,0 \\
\hline
\end{tabular}

Tabela 11: Pontos usados pelo MICTH interpolar a curva de magnetização.

\begin{tabular}{|c|c|c|c|}
\hline \multicolumn{2}{|c|}{ Transformador 4-7 } & \multicolumn{2}{c|}{ Transformador 5-6 } \\
\hline $\begin{array}{c}\text { Corrente de } \\
\text { Magnetização }\end{array}$ & $\begin{array}{c}\text { Fluxo de } \\
\text { Magnetização }\end{array}$ & $\begin{array}{c}\text { Corrente de } \\
\text { Magnetização }\end{array}$ & $\begin{array}{c}\text { Fluxo de } \\
\text { Magnetização }\end{array}$ \\
\hline 0,000 & 0,0000 & 0,000 & 0,0000 \\
\hline 0,050 & 0,0005 & 0,025 & 0,0003 \\
\hline 0,100 & 0,0011 & 0,075 & 0,0010 \\
\hline 0,150 & 0,0016 & 0,100 & 0,0013 \\
\hline 0,200 & 0,0019 & 0,200 & 0,0020 \\
\hline
\end{tabular}

Tabela 12: Pontos usados pelo ATP interpolar a curva de magnetização.

\begin{tabular}{|c|c|c|c|}
\hline \multicolumn{2}{|c|}{ Transformador 4-7 } & \multicolumn{2}{c|}{ Transformador 5-6 } \\
\hline $\begin{array}{c}\text { Corrente de } \\
\text { Magnetização }\end{array}$ & $\begin{array}{c}\text { Fluxo de } \\
\text { Magnetização }\end{array}$ & $\begin{array}{c}\text { Corrente de } \\
\text { Magnetização }\end{array}$ & $\begin{array}{c}\text { Fluxo de } \\
\text { Magnetização }\end{array}$ \\
\hline 0,000 & 0,0000 & 0,000 & 0,0000 \\
\hline 0,150 & 0,0016 & 0,100 & 0,0013 \\
\hline 0,200 & 0,0019 & 0,140 & 0,0018 \\
\hline 0,325 & 0,0021 & 0,200 & 0,0020 \\
\hline 0,510 & 0,0023 & 0,337 & 0,0022 \\
\hline 0,775 & 0,0025 & 0,500 & 0,0024 \\
\hline 1,250 & 0,0027 & 1,000 & 0,0027 \\
\hline 2,350 & 0,0029 & 1,875 & 0,0029 \\
\hline
\end{tabular}


O gerador conectado à barra 1 é equilibrado com fonte de tensão senoidal de módulo igual a 1,060p.u. e ângulo de $0^{\circ}$ na fase A. O gerador da barra 2 é do tipo PV com potência ativa especificada em 0,040p.u. e módulo da tensão igual a 1,045p.u. Devido às limitações do modelo do gerador utilizado no ATP em representar barras do tipo PV, este foi considerado como uma fonte de tensão com módulo e ângulo especificados a partir dos resultados obtidos pelo MICTH. Estes dados são apresentados na Tabela 13.

Tabela 13: Dados do gerador 2 (em p.u. e em ${ }^{\circ}$ ) usados pelo ATP.

\begin{tabular}{|c|c|c|c|c|c|c|}
\hline Sistema & \multicolumn{2}{|c|}{ Fase A } & \multicolumn{2}{c|}{ Fase B } & \multicolumn{2}{c|}{ Fase C } \\
\hline Teste & Mód. & Âng. & Mód. & Âng. & Mód. & Âng. \\
\hline $\begin{array}{c}\text { com } \\
\text { trafo } \\
\text { sem sa- } \\
\text { turação }\end{array}$ & 1,045 & $-5,957$ & 1,045 & $-130,1$ & 1,045 & 114,6 \\
\hline $\begin{array}{c}\text { com } \\
\text { trafo } \\
\text { saturado }\end{array}$ & 1,045 & $-5,298$ & 1,045 & $-129,2$ & 1,045 & $-115,0$ \\
\hline
\end{tabular}

Os parâmetros de linha são os mesmos apresentados em IEEE (1996). 